\title{
Antiplatelet regimens after ischemic stroke or transient ischemic attack: a systematic review and updated network meta-analysis
}

\author{
Seung Jin Jung ${ }^{1,2 \#}$, Bum Joon Kim ${ }^{3 \#}$, Chi Kyung Kim ${ }^{1,4}$, Sung Ryul Shim ${ }^{5,6}$, Jin-Man Jung ${ }^{1,7,8}$ \\ ${ }^{1}$ Department of Neurology, Korea University College of Medicine, Seoul, Republic of Korea; ${ }^{2}$ Department of Family Medicine, Gimpo Woori \\ Hospital, Gimpo, Republic of Korea; ${ }^{3}$ Department of Neurology, Asan Medical Center, University of Ulsan College of Medicine, Seoul, Republic \\ of Korea; ${ }^{4}$ Department of Neurology, Korea University Guro Hospital, Seoul, Republic of Korea; ${ }^{5}$ Department of Biomedical Informatics, CHA \\ University of Medicine, Seongnam, Republic of Korea; ${ }^{6}$ Institute for Biomedical Informatics, CHA University School of Medicine, CHA University, \\ Seongnam, Republic of Korea; ${ }^{7}$ Department of Neurology, Korea University Ansan Hospital, Ansan, Republic of Korea; ${ }^{8}$ Korea University Zebrafish \\ Translational Medical Research Center, Ansan, Republic of Korea \\ Contributions: (I) Conception and design: JM Jung; (II) Administrative support: JM Jung; (III) Provision of study materials or patients: SJ Jung, BJ \\ Kim, JM Jung; (IV) Collection and assembly of data: SJ Jung, BJ Kim, JM Jung; (V) Data analysis and interpretation: SR Shim; (VI) Manuscript \\ writing: All authors; (VII) Final approval of manuscript: All authors. \\ "These authors contributed equally to this work. \\ Correspondence to: Jin-Man Jung, MD, PhD. Department of Neurology, Korea University Ansan Hospital, 123, Jeokgeum-ro, Danwon-Gu, Ansan-Si, \\ Gyeonggi-Do 15355, South Korea. Email: dr.jinmanjung@gmail.com; Sung Ryul Shim, PhD. Department of Biomedical Informatics and Institute \\ for Biomedical Informatics, CHA University School of Medicine, 335, Pangyo-ro, Bundang-Gu, Seongnam-Si, Gyeonggi-Do 13488, Republic of \\ Korea. Email: ryul01@korea.ac.kr.
}

Background: It is still uncertain which antiplatelet regimen had the greatest net clinical benefit in patients who have suffered a transient ischemic attack or non-cardioembolic ischemic stroke, and it is necessary to choose the optimal regimen according to the clinical situation.

Methods: We utilized 3 databases of Medline, Embase, and the Cochrane Central Register of Controlled Trials to find randomized controlled trials that met our criteria, and performed network meta-analyses in recurrent stroke, composite outcomes, major bleeding events, recurrent ischemic stroke, and all bleeding events. Three-dimensional clustered rank plots were used to obtain the net clinical benefit. Subgroup analyses were performed according to the symptom-onset-to-treatment time ( $<72$ and $>72 \mathrm{~h}$ ), stroke subtypes (large artery atherosclerosis and small vessel occlusion), and dual antiplatelet agent treatment duration.

Results: A total of 69 trials were enrolled. Cilostazol was associated with a lower risk of recurrent stroke, major bleeding events, composite outcomes, recurrent ischemic stroke, and all bleeding events compared to low to medium dose aspirin. The three-dimensional rank plot showed that cilostazol had the highest net clinical benefit. The combination of aspirin plus clopidogrel had greater efficacy in the $<72 \mathrm{~h}$ after stroke onset and large artery atherosclerosis subgroups, and when it was restricted tol month of use major bleeding risk was not higher than aspirin. The combination of aspirin plus dipyridamole had greater efficacy and safety comparable to aspirin in terms of small vessel occlusion.

Conclusions: The efficacy and safety profiles among antiplatelet regimens may differ according to clinical situation, although cilostazol, aspirin plus clopidogrel, and aspirin plus dipyridamole may be considered as preferable options.

Keywords: Antiplatelet; network meta-analysis; ischemic stroke; transient ischemic attack

Submitted Jul 19, 2021. Accepted for publication Dec 24, 2021.

doi: $10.21037 / \mathrm{atm}-21-3748$

View this article at: https://dx.doi.org/10.21037/atm-21-3748 


\section{Introduction}

Non-cardioembolic ischemic stroke is an episode of neurologic dysfunction due to the permanent cerebral, retinal, or spinal infarction, caused by other than cardioembolism (1). Approximately $75 \%$ of ischemic stroke (IS) was attributed to the non-cardioembolism (2). Unlike IS, transient ischemic attack (TIA) is a brief and non-lasting focal neurological dysfunction, not result in the permanent infarction (3). Global prevalence of IS was 77.2 million people in 2019 (4), and the overall prevalence of TIA was about $2 \%(4,5)$. Patients with IS or TIA have a higher risk of recurrent stroke, with about $10-20 \%$ having a recurrent stroke within 3 months, most of which occur during the first few days (6-9).

The cornerstone for secondary non-cardioembolic ischemic stroke prevention is antiplatelet therapy, which includes aspirin (10-12). Although antiplatelet therapy reduces the incidence of atherosclerotic cardiovascular disease $(10,13)$, it also increases the risk of major bleeding such as intracranial hemorrhage and gastrointestinal bleeding requiring blood transfusions (13). Therefore, various antiplatelet agents and combinations of agents have been investigated to find regimens that demonstrate the best net clinical benefit regarding both potency and risk. However, as the number of available antiplatelet agents increase, it is becoming difficult to assess the effectiveness and safety of various treatment regimens directly. In the absence of direct comparisons, a network meta-analysis (NMA) can be a useful alternative to compare various antiplatelet treatment regimens (14-18). However, NMAs have historically had low clinical application for realworld clinicians since they have not considered several complicated conditions that patients may face.

The risk of stroke recurrence is fairly high during the first few days after an initial stroke or TIA $(6,9)$. Several trials (19-22) have suggested that intensive antiplatelet therapy should be administered as early as possible to reduce the high recurrence rate of acute stroke in that critical period. However, long-term intensive antiplatelet therapy failed to show benefit regarding the increased risk of bleeding. Therefore, the most appropriate antiplatelet regimen may differ depending on the time interval between symptom onset and treatment initiation. Moreover, since there are several studies $(21,23)$ that have demonstrated that limiting the duration of dual antiplatelet therapy (DAPT) is necessary to reduce the risk of major bleeding, DAPT treatment duration is an important safety consideration.
In addition, the most appropriate antiplatelet therapy may depend on the subtype of ischemic stroke (2). Lastly, the degree of thrombotic and bleeding tendency differs according to race and should also be considered $(24,25)$.

In this study, we performed an updated NMA, which included recently published randomized controlled trials (RCTs), to determine which antiplatelet regimen had the greatest net clinical benefit in terms of both efficacy and safety. This was determined through diverse subgroup analyses, which included the duration of DAPT treatment, the time interval between symptom onset and treatment initiation, and stroke subtypes [large artery atherosclerosis (LAA) and small vessel occlusion (SVO)].

We present the following article in accordance with the PRISMA NMA reporting checklist (26) (available at https:// atm.amegroups.com/article/view/10.21037/atm-21-3748/rc).

\section{Methods}

We registered this NMA to the International Prospective Register of Systematic Reviews (PROSPERO) in July 2020 (CRD42020186926).

\section{Search strategy}

We searched multiple comprehensive databases (Medline, Embase, and the Cochrane Central Register of Controlled Trials) for relevant studies from inception until November 9, 2020. The search terms were "ischemic stroke", "TIA", and "antiplatelet agents". Neither language nor the year of publication were restricted. A detailed search strategy, which was identical to that from a study of NMA (25), was presented in Table S1. The systematic search was performed by an independent collaborator (EJK). An additional manual search was conducted by two independent reviewers (SJJ and $\mathrm{JMJ}$ ) to prevent the omission of any relevant study. When necessary, the authors were contacted to obtain more data.

\section{Study selection}

We included all eligible studies that were randomized controlled trials that assessed the effectiveness or safety of antiplatelet regimens in TIA or non-cardioembolic ischemic stroke patients for the secondary prevention. Studies that involved cardiovascular or peripheral arterial diseases other than IS or TIA were excluded. However, for the CAPRIE (27) and CHARISMA (28) trials, we were 
able to extract the relevant outcomes from the IS or TIA patients through the subgroup analyses. While several trials had extended follow-up periods, only the results from the follow-up period included in the original study were analyzed. All disagreements concerning inclusion and exclusion were discussed until a consensus was reached.

\section{Data extraction and processing}

Data was extracted from the selected studies by two reviewers independently. A predefined data extraction template was used for the data extraction, and the following data were retrieved: baseline characteristics [age, sex, race, stroke subtypes, percentage of prior TIA, initial modified Rankin Scale (mRS) or National Institutes of Health Stroke Scale (NIHSS) score, and comorbidities including hypertension, diabetes, peripheral arterial disease, and myocardial infarction], study design (interventions, medication dosage, sample size, time interval between onset of symptoms and treatment initiation, treatment duration, and period of follow-up), and treatment outcome indicators, including the number of recurrent IS, recurrent stroke (ischemic + hemorrhagic stroke), recurrent TIA, recurrent myocardial infarction, vascular death, all-cause death, composite outcome (stroke, myocardial infarction, and vascular death), major bleeding event, and all bleeding events.

Primary efficacy and safety outcomes were recurrent stroke and major bleeding event respectively, and secondary outcomes were recurrent IS, composite outcomes, and all bleeding events. Major bleeding was defined as intracranial hemorrhage and extracranial bleeding requiring blood transfusions, which corresponds severe (life threatening) or moderate bleeding criteria in GUSTO classification (29). For trials that did not report outcomes, but whose outcomes could be obtained manually by adding or subtracting others, the value obtained manually was used as the number of outcomes. When we performed subgroup analyses using trials that did not include the relevant information, we obtained outcome data from the original study, from other sub-analyses $(30-32)$ or from meta-analyses $(33,34)$. In case of multi-arm trials, we took out only comparison arms that met inclusion criteria. The primary principle used to group antiplatelet regimens was the active ingredient of a specific drug. Different doses of the same active ingredient were grouped as the same antiplatelet regimen. However, since the purpose of a NMA was to assess the effectiveness and safety of the current standard antiplatelet therapy (low to medium dose aspirin) with other antiplatelet regimens, aspirin was grouped into three categories according to dosage [very low ( $<50 \mathrm{mg}$ ), low to medium (50-330 mg), and high dose $(>330 \mathrm{mg})]$. Low to medium dose aspirin was used as the common comparator.

\section{Quality assessment}

We assessed risks of bias of enrolled studies and the entire NMA using the Cochrane risk of bias assessment tool (35). Two reviewers evaluated seven domains of the risk of bias independently, and each domain was expressed as low risk, unclear risk, or high risk. Disagreement was resolved through discussion or adjudicated by a third reviewer when necessary.

\section{Statistical analysis}

We performed the NMA through the Bayesian method, using the R software "gemtc" package (36), and the relative risk ratio and $95 \%$ credible interval (CrI) were estimated. The counted number of patients in each study was subtracted based on the intention-to-treat population. Based on the outcomes mentioned previously, the efficacy and safety of different antiplatelet therapies were estimated. After a network setup, we performed Markov chain Monte Carlo simulation under the random effects model and performed a convergence diagnosis to choose the optimized network model (37). For the consistency test (37), we performed node-splitting methods to confirm that there is no statistical difference between direct and indirect evidence for a certain comparison. We also measured $\mathrm{I}^{2}$ values to consider the degree of inconsistency in the network as a whole (38). Publication bias was examined using funnel plots (39). We obtained the forest plot, league table, and the surface under the cumulative ranking curve (SUCRA) (40). The ranking of antiplatelet therapy regimens was presented using ranking probabilities. For assessing the efficacy and safety simultaneously, we made a three-dimensional clustered rank plot, which was obtained using SUCRA ranking probabilities in recurrent stroke, composite outcome, and major bleeding events.

We performed subgroup analyses for the time interval between onset of symptoms and treatment initiation $(<72$ and $>72 \mathrm{~h}$ ), the duration of DAPT treatment, and stroke subtypes (LAA and SVO). 


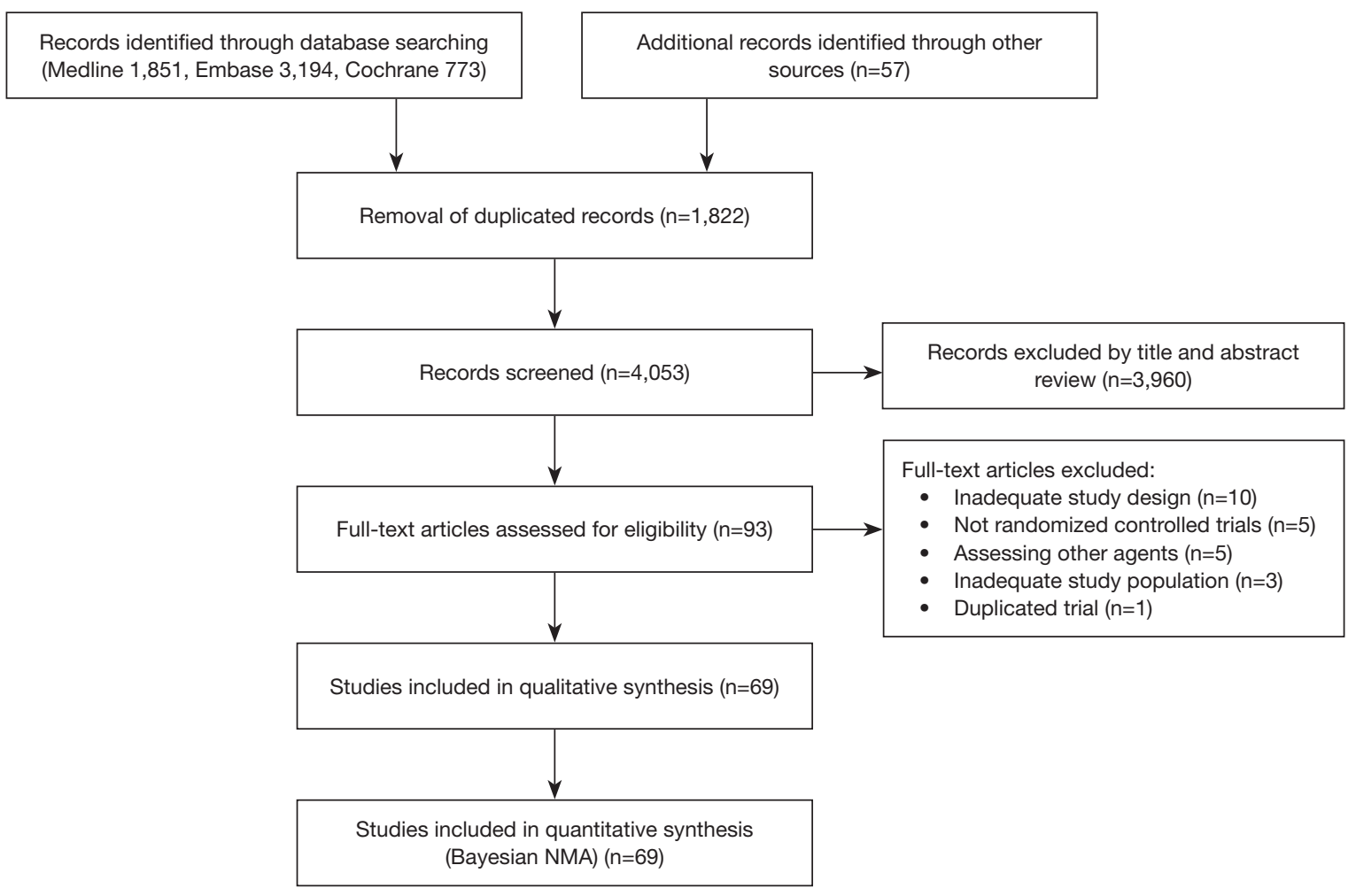

Figure 1 PRISMA flow chart.

\section{Results}

\section{Literature search}

Total 5,818 relevant studies (1,851 from Medline, 3,194 from Embase, and 773 from Cochrane Central Register of Controlled Trials) were initially detected. According to our inclusion and exclusion criteria, total 69 eligible articles published between March 1969 and November 2020 were incorporated into the NMA (Figure 1).

\section{Characteristics of the included studies}

The 69 included trials assessed 16 antiplatelet regimens including very low dose of aspirin (41), low to medium dose of aspirin [low dose (19-21,28,42-60); medium dose (27,41,61-72); low to medium dose (22,73-75)], high dose of aspirin (61,76-86), cilostazol $(48,50,52,59,68,87)$, clopidogrel (63,88-93), dipyridamole (94), prasugrel (93), sarpogrelate (49), ticagrelor (57), ticlopidine $(81,84,86,91,95-98)$, triflusal $(62,65,66,92)$, aspirin plus cilostazol $(47,54,55,69,75,99)$, aspirin plus clopidogrel $(19,21,22,28,45,46,51,56,58,70-72,74,90,99-101)$, aspirin plus dipyridamole $(20,43,53,67,73,79,82,85,89,90,102,103)$, aspirin plus ticagrelor $(60,101)$, and aspirin plus ticlopidine (98). Detailed characteristics of these 69 studies are exhibited in Table S2. Sixty-five trials were two-arm studies, and three trials $(58,79,90)$ had three intervention arms. One three-arm trial (58) compared two dosages of clopidogrel $(50 \mathrm{vs} .75 \mathrm{mg}$ ) in aspirin plus clopidogrel combination, and we merged the two intervention arms into an aspirin plus clopidogrel regimen and analyzed. Additionally, one trial (43) had four intervention arms.

At baseline, the patients were 65 years old on average. The mean prevalence of diabetes, hyperlipidemia, hypertension, and smoking was $28 \%, 40 \%, 64 \%$, and $38 \%$ respectively. The average period of follow-up was 15 months, while 14 trials had a duration of follow-up of one month $(44,46,56,60,71,90)$ or less $(27,45,51,64,72,85,95,102)$.

\section{Risk of bias}

The assessments of biases for all enrolled studies and the entire NMA were performed using the Cochrane Collaboration tool and we showed them in Figures S1,S2. 
A
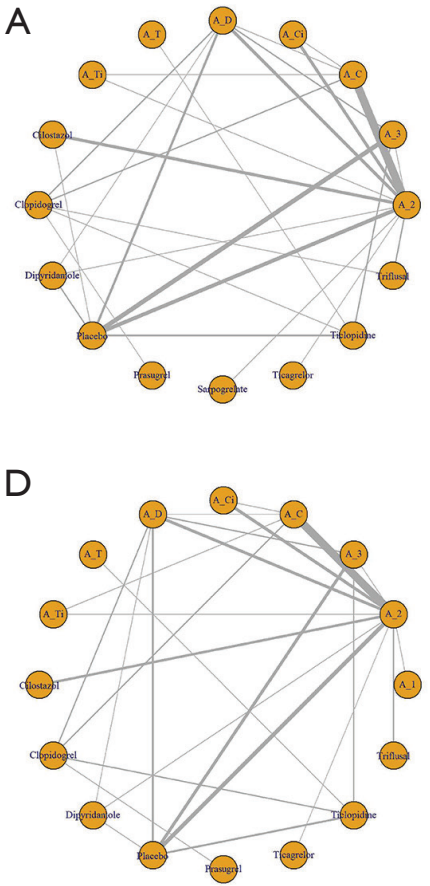

B

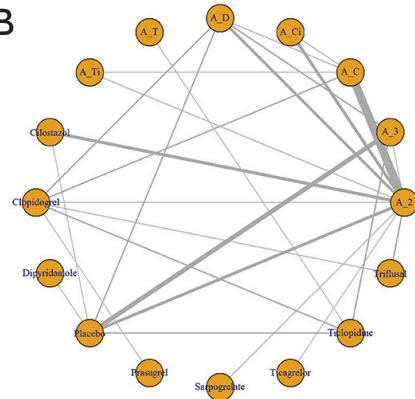

E

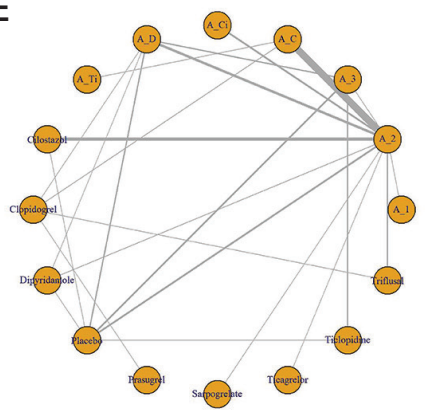

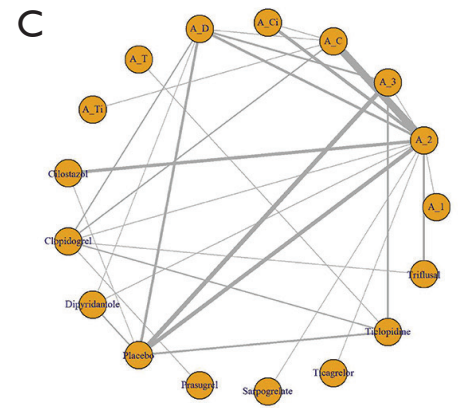

Figure 2 Network plots of the antiplatelet regimens. Antiplatelet treatment regimens are represented by nodes, and direct comparison trials between treatment regimens are linked with a line. The width of the line corresponds the sample size for each treatment regimen. (A) Recurrent stroke; (B) recurrent ischemic stroke; (C) composite outcomes; (D) major bleeding event; (E) all bleeding events. A_1, aspirin very low dose; A_2, aspirin low to medium dose; A_3, aspirin high dose; A_C, combination of aspirin plus clopidogrel; A_Ci, combination of aspirin plus cilostazol; A_D, combination of aspirin plus dipyridamole; A_T, combination of aspirin plus ticlopidine; A_Ti, combination of aspirin plus ticagrelor.

Most of the 69 studies were found to have a low risk of bias in the six domains. However, seven studies $(58,67,69,81,85,90,98)$ did not describe the randomization methods in detail. The CAPRIE (27) and the CHARISMA studies (28) were subgroup analyses. The procedures, utilized to blind the participants and staffs or the outcome assessments, were not described in ten studies $(46,50,55,58$, $69,71,74,85,90,98)$. Five studies $(20,72,73,75,92)$ were openlabel trials. And five studies $(27,51,64,67,101)$ were assessormasked open-label trials.

\section{Outcomes of interest}

Figure 2 depicts the network plots for each outcome. We displayed the main results of the NMA in Figure 3, Tables S3-S8, and Figure S3.

\section{Recurrent stroke}

Sixty-three trials reported recurrent stroke events with a total sample size of 159,461 . Cilostazol, aspirin plus clopidogrel, aspirin plus ticagrelor, clopidogrel, and aspirin plus dipyridamole had a decreased risk of recurrent stroke to aspirin alone. Cilostazol has the highest SUCRA value, the combination of aspirin plus clopidogrel had the second highest, and the combination of aspirin plus ticagrelor had the third highest value.

\section{Recurrent IS}

Sixty-two trials with a sample size of 160,772 reported recurrent IS. Aspirin plus clopidogrel, cilostazol, aspirin plus ticagrelor, aspirin plus dipyridamole, and clopidogrel alone had a decreased risk of recurrent IS to aspirin alone. The combination of aspirin plus clopidogrel had the highest SUCRA value, cilostazol had the second highest, and the combination of aspirin plus ticagrelor had the third highest value.

\section{Composite outcomes}

Sixty-six trials reported composite outcomes with a sample size of 160,344. Aspirin plus ticagrelor, cilostazol, aspirin 


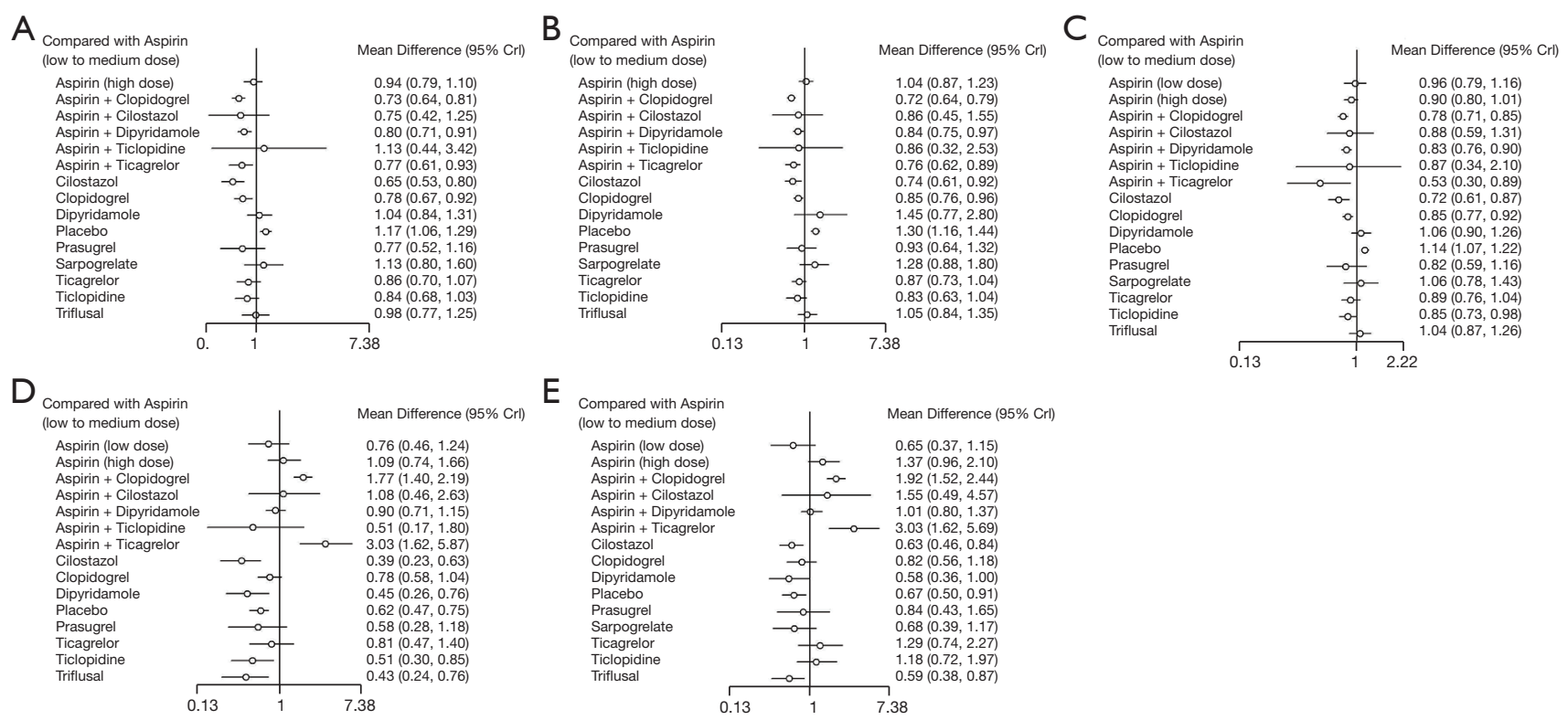

Figure 3 Forrest plots of the antiplatelet regimens compared to aspirin (low to medium dose). (A) Recurrent stroke; (B) recurrent ischemic stroke; (C) composite outcomes; (D) major bleeding event; (E) all bleeding events.

plus clopidogrel, aspirin plus dipyridamole, and clopidogrel had a decreased risk of composite outcomes to aspirin alone. The combination of aspirin plus ticagrelor had the highest SUCRA value, cilostazol had the second highest, and the combination of aspirin plus clopidogrel had the third highest value.

\section{Major bleeding event}

Fifty-seven trials reported major bleeding with a sample size of 156,436. Compared to aspirin, cilostazol, triflusal, dipyridamole, and ticlopidine were associated with a lower risk of major bleeding. However, aspirin plus ticagrelor and aspirin plus clopidogrel had an increased risk of major bleeding events to aspirin alone. Cilostazol had the highest SUCRA value and aspirin plus ticagrelor had the lowest value.

\section{All bleeding events}

Forty-three trials reported bleeding with a sample size of 102,102. Triflusal and cilostazol had a decreased risk of all bleeding events to aspirin alone. However, aspirin plus ticagrelor and aspirin plus clopidogrel had an increased risk of all bleeding events to aspirin. Triflusal had the highest SUCRA value, cilostazol had the third highest, and aspirin plus ticagrelor had the lowest value.

\section{Net clinical benefit}

We supposed that the antiplatelet regimen, which had the high probabilities of first ranking in efficacy (recurrent stroke and composite outcomes) and safety (major bleeding events) simultaneously, had the greatest net clinical benefit. As shown at Figure 4, cilostazol had the greatest net clinical benefit.

\section{Consistency assessment and publication bias}

Figure S4 shows the results of node-splitting assessments. There were no loop inconsistencies when comparing effect estimates for all outcomes measured based on direct $v s$. indirect evidence (all $\mathrm{P}>0.05$ ). In addition, very low heterogeneity was noticed when assessing inconsistency across trials (all $\left.\mathrm{I}^{2}<5 \%\right)$. The symmetry of the comparisonadjusted funnel plots indicated that there was no evidence of publication bias (Figure S5).

\section{Subgroup analyses}

Time interval from stroke onset to treatment initiation $<72$ hours

Twenty-six studies (19-22,27,31,33,44,51,56,57,60,64,67$69,71,72,74,75,85,101)$ were included in this subgroup 


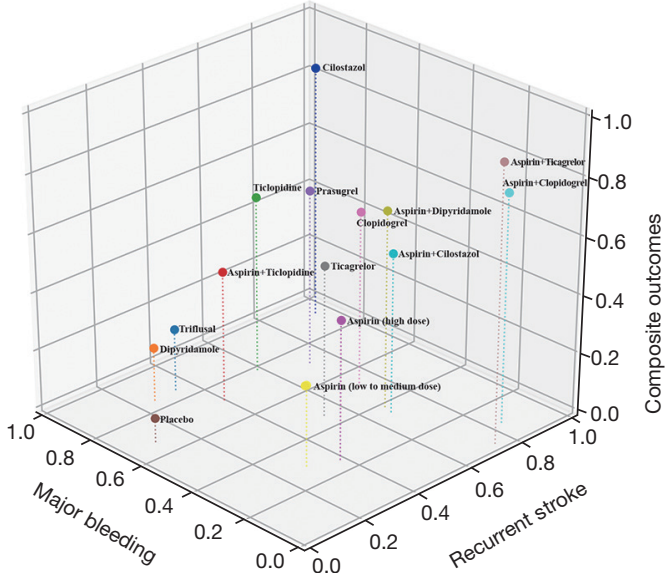

Figure 4 Three-dimensional clustered rank plot. Values of each axis are the probabilities which antiplatelet regimens had the first ranking for recurrent stroke, composite outcomes, and major bleeding event using values of surface under the cumulative ranking curve. The point $(1,1,1)$ is the hypothetical point with $100 \%$ probability of first ranking for all interesting events. Since cilostazol is the closest antiplatelet therapy regimen to the point (1, $1,1)$, it was considered to have the greatest net clinical benefit.

analysis. The total sample size was 83,306 and the mean follow-up period was 2.3 months. Among included studies, outcome data from one subgroup or post hoc analysis (31) and one meta-analysis (33) were used. The overall heterogeneity was low (all $\mathrm{I}^{2}<5 \%$ ), and no inconsistency was noticed except at composite outcomes. The node-splitting assessment was not evaluated in the analysis of composite outcomes because of a lack of outcome data. Four DAPT treatments (aspirin plus clopidogrel, aspirin plus ticagrelor, aspirin plus cilostazol, and aspirin plus dipyridamole) and four monotherapies (low to medium dose aspirin, high dose aspirin, cilostazol, and ticagrelor) were compared (Figure S6). The combination of aspirin plus clopidogrel had decreased risks of recurrent stroke, recurrent IS, and composite outcomes to aspirin alone. The combination of aspirin plus ticagrelor had decreased risks of recurrent IS and composite outcomes to aspirin alone. Although cilostazol ranked high, it was not associated with better efficacy than aspirin. Safety in terms of all bleeding events was not obtained due to a lack of outcome data. Two DAPT combinations (aspirin plus ticagrelor and aspirin plus clopidogrel) had an increased risk of major bleeding events to aspirin alone. Other regimens did not have a higher risk of major bleeding than aspirin.
Time interval from stroke onset to treatment initiation $>72$ hours

Forty-nine studies were included with a sample size of 90,665 (Table S2). None of the included studies limited or included only patients who received treatment after 72 $\mathrm{h}$ of symptom onset. However, majority of index events for enrollment occurred $72 \mathrm{~h}$ following the beginning of symptoms, and most trials included a three-month or longer follow-up period, which could reflect the chronic and stable stage after the index event. No inconsistency was observed in node-splitting assessments (all $\mathrm{P}>0.05$ ), and an overall heterogeneity in the network was low (all $\mathrm{I}^{2}<8 \%$ ).

Cilostazol, aspirin plus clopidogrel, clopidogrel, and aspirin plus dipyridamole had decreased risks of recurrent stroke, recurrent IS, and composite outcomes to aspirin alone (Figure S7). Cilostazol had the highest SUCRA values for recurrent stroke and composite outcomes. Cilostazol had also decreased risks of major bleeding event and all bleeding events compared to aspirin alone, and it had the second highest SUCRA value for major and all bleeding events. Although aspirin plus clopidogrel had the highest SUCRA value for recurrent IS, it was the only regimen that had a higher risk of major and all bleeding events than aspirin, and its SUCRA ranks were the lowest.

\section{Stroke subtype-LAA}

Ten studies $(30,32,45,47,51,55,56,58,71,99)$ were included with a sample size of 3,092 . The outcome data from two sub-analyses $(30,32)$, which had analyzed only patients with LAA in the original trials, were used. There was no inconsistency in node-splitting assessments (all $\mathrm{P}>0.05$ ), and no heterogeneity in the network (all $\mathrm{I}^{2}=0 \%$ ). The antiplatelet regimens included two DAPTs (aspirin plus clopidogrel and aspirin plus cilostazol), low to medium dose aspirin, and cilostazol (Figure S8). The combination of aspirin plus clopidogrel had decreased risks of recurrent stroke, recurrent IS, and composite outcomes to aspirin alone. In terms of safety, the combination of aspirin plus clopidogrel had increased risks of major bleeding event and all bleeding events compared to aspirin alone. Cilostazol was analyzed only in terms of recurrent stroke and ischemic stroke due to a lack of other outcome data.

\section{Stroke subtype-SVO}

Five studies $(30,32,34,54,70)$ with the total sample size of 32,690 were used as outcome extraction sources of 13 included trials $(43,44,52,54,70,73,79,86,87,89,91,97,100)$. Two trials investigated the comparative effects of antiplatelet 
regimens in only patients with SVO $(54,70)$. Outcome data of 2 trials from subgroup or post hoc analyses $(30,32)$ and 9 trials from one meta-analysis (34) which had been extracted and pooled in only patients with SVO of the original trial population, were included. The overall heterogeneity was low (all $\mathrm{I}^{2}<8 \%$ ), and no inconsistency was observed in the analyses of IS and composite outcomes (all $\mathrm{P}>0.05$ ). The node-splitting assessment was not performed in the analysis of recurrent stroke because of a lack of outcome data. Three DAPTs (aspirin plus cilostazol, aspirin plus clopidogrel, and aspirin plus dipyridamole) as well as ticlopidine, clopidogrel, cilostazol, low to medium dose aspirin, and dipyridamole were included (Figure S9). Bleeding outcomes were not analyzed due to the absence of data. All included antiplatelet regimens were comparable to aspirin in terms of recurrent stroke, recurrent IS, and composite outcomes. However, the combination of aspirin plus dipyridamole was associated with the highest SUCRA value for recurrent IS and composite outcomes, and the second highest for recurrent stroke.

\section{Treatment duration of DAPT}

All 69 trials were included with the total sample size of 171,595 . The overall heterogeneity was low (all $\mathrm{I}^{2}<5 \%$ ), and there was no inconsistency in node-splitting assessments except at aspirin $v s$. aspirin plus clopidogrel $(\mathrm{P}=0.02)$ and aspirin plus clopidogrel $v s$. aspirin plus ticagrelor $(\mathrm{P}=0.02)$. Combination regimens including aspirin plus cilostazol, aspirin plus clopidogrel, and aspirin plus dipyridamole were divided according to DAPT treatment duration $(<$ or $>1$ month). As shown in Figure S10, aspirin plus clopidogrel ( $<1$ month) had greater efficacy with preserved safety compared to both aspirin plus clopidogrel ( $>1$ month) and aspirin alone. However, the major bleeding risk associated with aspirin plus clopidogrel ( $>1$ month) was higher than that with aspirin. The combination of aspirin plus cilostazol had comparable efficacy and safety to aspirin regardless of the duration of therapy. For the combination of aspirin plus dipyridamole, however, only a duration $>1$ month had greater efficacy for recurrent stroke, recurrent IS, and composite outcomes, and safety similar to aspirin.

\section{Discussion}

Our Bayesian NMA showed that cilostazol had the highest net clinical benefit and decreased risks of recurrent stroke, recurrent IS, composite outcomes, major bleeding event, and all bleeding events to aspirin alone. This finding was similar with conclusions of previous NMAs, but we revealed additionally that several regimens have different efficacy and safety profiles according to the treatment initiation, stroke subtype, and DAPT treatment duration. Most of all, cilostazol was found to have the highest net clinical benefit especially in the $>72$ hours from stroke onset subgroup analysis. And the combination of aspirin plus clopidogrel had a higher efficacy than aspirin and a high SUCRA value in the $<72$ hours from stroke onset subgroup analysis, and in the DAPT duration subgroup analysis, the major bleeding risk of the $<1$ month group was not higher than that of aspirin. Also, it had higher efficacy without increasing major bleeding risk compared to aspirin in the LAA subgroup analysis. In the case of SVO, there was no antiplatelet regimen with higher efficacy than aspirin, but in the SUCRA ranking, the combination of aspirin plus dipyridamole had the highest value. Therefore, efficacy and safety profiles among antiplatelet regimens may differ according to the clinical situation, stroke subtypes, and treatment duration of dual antiplatelet agents.

Cilostazol was found to have the potential to be a first option for the secondary prevention of subacute and chronic stroke. The high efficacy and safety of cilostazol were due to the lower risk of bleeding and pleiotropic mechanisms in the vascular endothelium associated with phosphodiesterase type-3 (PDE3) inhibitors (104). However, cilostazol has been studied mainly in Asian populations, and evidence has shown a higher bleeding tendency (105) and a difference in the mechanism of stroke (106) in Asians compared to nonAsians. Therefore, the high net clinical benefit may be more prominent in Asians (25), and it is necessary to evaluate the efficacy and safety of cilostazol in non-Asian populations. In other hands, although cilostazol did not have a higher efficacy than aspirin in the subgroup analysis of stroke onset to treatment initiation time interval $<72 \mathrm{~h}$, there is a need for further evaluation of the role of cilostazol in the acute phase of stroke since its SUCRA value was ranked highly. In addition, more clinical evidence concerning the combination of aspirin and cilostazol is needed because a recent study (107), which reported that cilostazol-based DAPT was beneficial and safe, was excluded from this NMA for its unique study design which cannot be analyzed in this NMA. There is some evidence for the clinical applicability of aspirin plus cilostazol since superior efficacy and safety was seen with aspirin plus dipyridamole, which has a similar mechanism of action to that of cilostazol after longer treatment compared to aspirin alone.

The combination of aspirin plus clopidogrel was found 
to be the optimal antiplatelet regimen in patients within the acute period of stroke because it had a higher efficacy than aspirin and a high SUCRA value in the $<72$ hours from stroke onset subgroup analysis. Although aspirin plus clopidogrel had a higher bleeding risk, if the increased bleeding risk was not higher than the high recurrence rate in the acute period of stroke, it may have a positive net benefit. In addition, in the DAPT duration subgroup analysis, the major bleeding risk of the $<1$ month group was not higher than that of aspirin; therefore, the use of aspirin plus clopidogrel for $<1$ month is thought to have a clear positive net clinical benefit. However, since patients with a minor stroke or TIA were mainly included in the RCTs using DAPT during the acute phase, the low risk for intracerebral hemorrhage should be carefully interpreted.

The combination of aspirin plus ticagrelor showed almost similar efficacy and safety profiles to the combination of aspirin plus clopidogrel, but unlike aspirin plus clopidogrel, the risk of major bleeding with aspirin plus ticagrelor was higher than that with aspirin, even though it was administered for $<1$ month. However, it is worth noting that aspirin plus ticagrelor had the highest SUCRA value in composite outcomes, although it ranked third in recurrent stroke. This finding suggests that the prevention of cardiovascular events with the combination of aspirin plus ticagrelor is considerably greater in patients with IS. Therefore, we recommend the combination of aspirin plus ticagrelor in patients with a high risk of cardiovascular events after IS.

In case of SVO, the combination of aspirin plus dipyridamole had the highest SUCRA value. It is thought that dipyridamole played a role in SVO prevention as a vasodilator. It is important to note that cilostazol, which had high SUCRA values throughout this NMA, did not rank high in the SVO subgroup analysis. However, of the RCTs included, only a few were conducted on SVO. Therefore, in this subgroup analysis, a lot of data were from sub-analyses and meta-analyses, which may have increased the likelihood of a bias. More clinical trials regarding SVO are needed for a definite conclusion.

This study has several limitations. Although there was no evidence of inconsistency in the entire NMA, some node-splitting assessments were not evaluated in the subgroup analyses because of a lack of outcome data. And there was an inconsistency in two loops (aspirin $v s$. aspirin plus clopidogrel, and aspirin plus clopidogrel $v s$. aspirin plus ticagrelor) in recurrent IS of the DAPT duration subgroup analysis. However, it is possible that one or a few may show inconsistency by chance when multiple loops are tested for inconsistency (26), and since this is not related to differences according to the DAPT duration, we determined it would not affect the overall conclusion. Likewise, though inconsistency tests were not conducted in some subgroup analyses, these were largely irrelevant to our main conclusions. Second, in subgroup analyses, some among outcome data were extracted from post hoc analyses or meta-analyses due to the difficulty of data assess. Thus, there is a possibility of randomization error and bias in selective reporting. Third, there is a possibility of design inconsistency. We included 69 trials and the total 171,595 patients. Although the overall heterogeneity in the whole network was very low, the conclusions of each subgroup analysis were somewhat different from that of the entire NMA. So, there may be another unexpected variable that could affect the NMA conclusions. For example, some studies focused patients with minor stroke or high-risk TIA.

In conclusion, efficacy and safety profiles among antiplatelet regimens may differ according to the clinical situation, stroke subtypes, and treatment duration of dual antiplatelet agents although cilostazol, aspirin plus clopidogrel, and aspirin plus dipyridamole were considered as preferable options in patients with non-cardioembolic stroke or TIA. More randomized investigations are therefore needed to identify the most suitable antiplatelet therapy regimens for different clinical conditions and stroke subtypes, particularly SVO.

\section{Acknowledgments}

The authors would like to thank Eun-Ji Kang from the Medical Library, Korea University, for searching abstracts and articles related to this study.

Funding: This work was supported by the Korea Medical Device Development Fund grant funded by the Korea government (the Ministry of Science and ICT, the Ministry of Trade, Industry and Energy, the Ministry of Health \& Welfare, the Ministry of Food and Drug Safety) (Project Number: 9991007421, KMDF_PR_20200901_0187).

\section{Footnote}

Reporting Checklist: The authors have completed the PRISMA NMA reporting checklist. Available at https:// atm.amegroups.com/article/view/10.21037/atm-21-3748/rc

Conflicts of Interest: All authors have completed the ICMJE 
uniform disclosure form (available at https://atm.amegroups. com/article/view/10.21037/atm-21-3748/coif). JMJ serves as an unpaid editorial board member of Annals of Translational Medicine from November 2019 to October 2021. JMJ has received the following: lecture honoraria from Pfizer, SanofiAventis, Otsuka, and Hanmi Pharmaceutical Co., Ltd.; consulting fees from Daewoong Pharmaceutical Co., Ltd. JMJ reports payments which were made to his institution: National Research Foundation of Korea (NRF) funded by the Ministry of Science and ICT; Ansan-Si hidden champion fostering and supporting project funded by Ansan city. The other authors have no conflicts of interest to declare.

Ethical Statement: The authors are accountable for all aspects of the work in ensuring that questions related to the accuracy or integrity of any part of the work are appropriately investigated and resolved.

Open Access Statement: This is an Open Access article distributed in accordance with the Creative Commons Attribution-NonCommercial-NoDerivs 4.0 International License (CC BY-NC-ND 4.0), which permits the noncommercial replication and distribution of the article with the strict proviso that no changes or edits are made and the original work is properly cited (including links to both the formal publication through the relevant DOI and the license). See: https://creativecommons.org/licenses/by-nc-nd/4.0/.

\section{References}

1. Adams HP Jr, Bendixen BH, Kappelle LJ, et al. Classification of subtype of acute ischemic stroke. Definitions for use in a multicenter clinical trial. TOAST. Trial of Org 10172 in Acute Stroke Treatment. Stroke 1993;24:35-41.

2. Del Brutto VJ, Chaturvedi S, Diener HC, et al. Antithrombotic Therapy to Prevent Recurrent Strokes in Ischemic Cerebrovascular Disease: JACC Scientific Expert Panel. J Am Coll Cardiol 2019;74:786-803.

3. Easton JD, Saver JL, Albers GW, et al. Definition and Evaluation of Transient Ischemic Attack. Stroke 2009;40:2276-93.

4. Virani SS, Alonso A, Aparicio HJ, et al. Heart Disease and Stroke Statistics-2021 Update: A Report From the American Heart Association. Circulation 2021;143:e254-743.

5. Wang Y, Zhao X, Jiang Y, et al. Prevalence, knowledge, and treatment of transient ischemic attacks in China.
Neurology 2015;84:2354-61.

6. Johnston SC, Gress DR, Browner WS, et al. Short-term prognosis after emergency department diagnosis of TIA. JAMA 2000;284:2901-6.

7. Coull AJ, Lovett JK, Rothwell PM, et al. Population based study of early risk of stroke after transient ischaemic attack or minor stroke: implications for public education and organisation of services. BMJ 2004;328:326.

8. Rothwell PM, Giles MF, Chandratheva A, et al. Effect of urgent treatment of transient ischaemic attack and minor stroke on early recurrent stroke (EXPRESS study): a prospective population-based sequential comparison. Lancet 2007;370:1432-42.

9. Giles MF, Rothwell PM. Risk of stroke early after transient ischaemic attack: a systematic review and meta-analysis. Lancet Neurol 2007;6:1063-72.

10. Antiplatelet Trialists' Collaboration. Collaborative overview of randomised trials of antiplatelet therapy--I: Prevention of death, myocardial infarction, and stroke by prolonged antiplatelet therapy in various categories of patients. BMJ 1994;308:81-106.

11. Antithrombotic Trialists' (ATT) Collaboration; Baigent C, Blackwell L, et al. Aspirin in the primary and secondary prevention of vascular disease: collaborative meta-analysis of individual participant data from randomised trials. Lancet 2009;373:1849-60.

12. Algra A, van Gijn J. Cumulative meta-analysis of aspirin efficacy after cerebral ischaemia of arterial origin. J Neurol Neurosurg Psychiatry 1999;66:255.

13. O'Donnell MJ, Hankey GJ, Eikelboom JW. Antiplatelet therapy for secondary prevention of noncardioembolic ischemic stroke: a critical review. Stroke 2008;39:1638-46.

14. Xie W, Zheng F, Zhong B, et al. Long-Term Antiplatelet Mono- and Dual Therapies After Ischemic Stroke or Transient Ischemic Attack: Network Meta-Analysis. J Am Heart Assoc 2015;4:e002259.

15. Niu PP, Guo ZN, Jin H, et al. Antiplatelet regimens in the long-term secondary prevention of transient ischaemic attack and ischaemic stroke: an updated network metaanalysis. BMJ Open 2016;6:e009013.

16. Wang W, Zhang L, Liu W, et al. Antiplatelet Agents for the Secondary Prevention of Ischemic Stroke or Transient Ischemic Attack: A Network Meta-Analysis. J Stroke Cerebrovasc Dis 2016;25:1081-9.

17. Huang HP, Lin WH, Chen SG, et al. Comparative Efficacy and Safety of Nine Anti-Platelet Therapies for Patients with Ischemic Stroke or Transient Ischemic Attack: a Mixed Treatment Comparisons. Mol Neurobiol 
2017;54:1456-66.

18. Xiang RW, Han RB, Yang JY, et al. Comparative efficacy and safety of multiple antiplatelet therapies for secondary prevention of ischemic stroke or transient ischemic attack: A network meta-analysis. J Neurol Sci 2019;399:199-206.

19. Kennedy J, Hill MD, Ryckborst KJ, et al. Fast assessment of stroke and transient ischaemic attack to prevent early recurrence (FASTER): a randomised controlled pilot trial. Lancet Neurol 2007;6:961-9.

20. Dengler R, Diener HC, Schwartz A, et al. Early treatment with aspirin plus extended-release dipyridamole for transient ischaemic attack or ischaemic stroke within $24 \mathrm{~h}$ of symptom onset (EARLY trial): a randomised, open-label, blinded-endpoint trial. Lancet Neurol 2010;9:159-66.

21. Wang Y, Wang Y, Zhao X, et al. Clopidogrel with aspirin in acute minor stroke or transient ischemic attack. N Engl J Med 2013;369:11-9.

22. Johnston SC, Easton JD, Farrant M, et al. Clopidogrel and Aspirin in Acute Ischemic Stroke and High-Risk TIA. N Engl J Med 2018;379:215-25.

23. Johnston SC, Elm JJ, Easton JD, et al. Time Course for Benefit and Risk of Clopidogrel and Aspirin After Acute Transient Ischemic Attack and Minor Ischemic Stroke. Circulation 2019;140:658-64.

24. Cho H, Kang J, Kim HS, et al. Ethnic Differences in Oral Antithrombotic Therapy. Korean Circ J 2020;50:645-57.

25. Jung SJ, Shim SR, Kim BJ, et al. Antiplatelet regimens for Asian patients with ischemic stroke or transient ischemic attack: a systematic review and network meta-analysis. Ann Transl Med 2021;9:753.

26. Hutton B, Salanti G, Caldwell DM, et al. The PRISMA extension statement for reporting of systematic reviews incorporating network meta-analyses of health care interventions: checklist and explanations. Ann Intern Med 2015;162:777-84.

27. Multicentre Acute Stroke Trial-Italy (MAST-I) Group. Randomised controlled trial of streptokinase, aspirin, and combination of both in treatment of acute ischaemic stroke. Lancet 1995;346:1509-14.

28. Hankey GJ, Johnston SC, Easton JD, et al. Effect of clopidogrel plus ASA vs. ASA early after TIA and ischaemic stroke: a substudy of the CHARISMA trial. Int J Stroke 2011;6:3-9.

29. GUSTO investigators. An international randomized trial comparing four thrombolytic strategies for acute myocardial infarction. N Engl J Med 1993;329:673-82.

30. Matsumoto M. Cilostazol in secondary prevention of stroke: impact of the Cilostazol Stroke Prevention Study.
Atheroscler Suppl 2005;6:33-40.

31. Bath PM, Cotton D, Martin RH, et al. Effect of combined aspirin and extended-release dipyridamole versus clopidogrel on functional outcome and recurrence in acute, mild ischemic stroke: PRoFESS subgroup analysis. Stroke 2010;41:732-8.

32. Uchiyama S, Shinohara Y, Katayama Y, et al. Benefit of cilostazol in patients with high risk of bleeding: subanalysis of cilostazol stroke prevention study 2. Cerebrovasc Dis 2014;37:296-303.

33. Wong KS, Wang Y, Leng X, et al. Early dual versus mono antiplatelet therapy for acute non-cardioembolic ischemic stroke or transient ischemic attack: an updated systematic review and meta-analysis. Circulation 2013;128:1656-66.

34. Kwok CS, Shoamanesh A, Copley HC, et al. Efficacy of Antiplatelet Therapy in Secondary Prevention Following Lacunar Stroke. Stroke 2015;46:1014-23.

35. Higgins JP, Thomas J, Chandler J, et al. Cochrane handbook for systematic reviews of interventions. 2nd ed. Chichester (UK): John Wiley \& Sons; 2019.

36. GitHub. R software "gemtc" \& "netmeta" packages. Available online: https://github.com/gertvv/gemtc. Accessed July 102021.

37. Shim SR, Kim SJ, Lee J, et al. Network meta-analysis: application and practice using $\mathrm{R}$ software. Epidemiol Health 2019;41:e2019013.

38. Higgins JP, Thompson SG, Deeks JJ, et al. Measuring inconsistency in meta-analyses. BMJ 2003;327:557-60.

39. Chaimani A, Higgins JP, Mavridis D, et al. Graphical tools for network meta-analysis in STATA. PLoS One 2013;8:e76654.

40. Salanti G, Ades AE, Ioannidis JP. Graphical methods and numerical summaries for presenting results from multipletreatment meta-analysis: an overview and tutorial. J Clin Epidemiol 2011;64:163-71.

41. Dutch TIA Trial Study Group; van Gijn J, Algra A, et al. A comparison of two doses of aspirin $(30 \mathrm{mg}$ vs. $283 \mathrm{mg}$ a day) in patients after a transient ischemic attack or minor ischemic stroke. N Engl J Med 1991;325:1261-6.

42. Swedish Aspirin Low-Dose Trial (SALT) of $75 \mathrm{mg}$ aspirin as secondary prophylaxis after cerebrovascular ischaemic events. The SALT Collaborative Group. Lancet 1991;338:1345-9.

43. Diener HC, Cunha L, Forbes C, et al. European Stroke Prevention Study. 2. Dipyridamole and acetylsalicylic acid in the secondary prevention of stroke. J Neurol Sci 1996;143:1-13.

44. CAST: randomised placebo-controlled trial of early aspirin 
use in 20000 patients with acute ischaemic stroke. Lancet 1997;349:1641-9.

45. Markus HS, Droste DW, Kaps M, et al. Dual Antiplatelet Therapy With Clopidogrel and Aspirin in Symptomatic Carotid Stenosis Evaluated Using Doppler Embolic Signal Detection. Circulation 2005;111:2233-40.

46. Serebruany VL, Malinin AI, Ziai W, et al. Effects of clopidogrel and aspirin in combination versus aspirin alone on platelet activation and major receptor expression in patients after recent ischemic stroke: for the Plavix Use for Treatment of Stroke (PLUTO-Stroke) trial. Stroke 2005;36:2289-92.

47. Kwon SU, Cho YJ, Koo JS, et al. Cilostazol prevents the progression of the symptomatic intracranial arterial stenosis: the multicenter double-blind placebo-controlled trial of cilostazol in symptomatic intracranial arterial stenosis. Stroke 2005;36:782-6.

48. Huang Y, Cheng Y, Wu J, et al. Cilostazol as an alternative to aspirin after ischaemic stroke: a randomised, doubleblind, pilot study. Lancet Neurol 2008;7:494-9.

49. Shinohara Y, Nishimaru K, Sawada T, et al. SarpogrelateAspirin Comparative Clinical Study for Efficacy and Safety in Secondary Prevention of Cerebral Infarction (S-ACCESS): A randomized, double-blind, aspirincontrolled trial. Stroke 2008;39:1827-33.

50. Guo JJ, Xu E, Lin QY, et al. Effect of cilostazol on cerebral arteries in secondary prevention of ischemic stroke. Neurosci Bull 2009;25:383-90.

51. Wong KS, Chen C, Fu J, et al. Clopidogrel plus aspirin versus aspirin alone for reducing embolisation in patients with acute symptomatic cerebral or carotid artery stenosis (CLAIR study): a randomised, open-label, blindedendpoint trial. Lancet Neurol 2010;9:489-97.

52. Shinohara Y, Katayama Y, Uchiyama S, et al. Cilostazol for prevention of secondary stroke (CSPS 2): an aspirincontrolled, double-blind, randomised non-inferiority trial. Lancet Neurol 2010;9:959-68.

53. Uchiyama S, Ikeda Y, Urano Y, et al. The Japanese aggrenox (extended-release dipyridamole plus aspirin) stroke prevention versus aspirin programme (JASAP) study: a randomized, double-blind, controlled trial. Cerebrovasc Dis 2011;31:601-13.

54. Han SW, Lee SS, Kim SH, et al. Effect of cilostazol in acute lacunar infarction based on pulsatility index of transcranial Doppler (ECLIPse): a multicenter, randomized, double-blind, placebo-controlled trial. Eur Neurol 2013;69:33-40.

55. Uchiyama S, Sakai N, Toi S, et al. Final Results of
Cilostazol-Aspirin Therapy against Recurrent Stroke with Intracranial Artery Stenosis (CATHARSIS). Cerebrovasc Dis Extra 2015;5:1-13.

56. Hong KS, Lee SH, Kim EG, et al. Recurrent Ischemic Lesions After Acute Atherothrombotic Stroke: Clopidogrel Plus Aspirin Versus Aspirin Alone. Stroke 2016;47:2323-30.

57. Johnston SC, Amarenco P, Albers GW, et al. Ticagrelor versus Aspirin in Acute Stroke or Transient Ischemic Attack. N Engl J Med 2016;375:35-43.

58. Zuo FT, Liu H, Wu HJ, et al. The effectiveness and safety of dual antiplatelet therapy in ischemic cerebrovascular disease with intracranial and extracranial arteriostenosis in Chinese patients: A randomized and controlled trail. Medicine (Baltimore) 2017;96:e5497.

59. Kim BJ, Lee EJ, Kwon SU, et al. Prevention of cardiovascular events in Asian patients with ischaemic stroke at high risk of cerebral haemorrhage (PICASSO): a multicentre, randomised controlled trial. Lancet Neurol 2018;17:509-18.

60. Johnston SC, Amarenco P, Denison H, et al. Ticagrelor and Aspirin or Aspirin Alone in Acute Ischemic Stroke or TIA. N Engl J Med 2020;383:207-17.

61. Farrell B, Godwin J, Richards S, et al. The United Kingdom transient ischaemic attack (UK-TIA) aspirin trial: final results. J Neurol Neurosurg Psychiatry 1991;54:1044-54.

62. Smirne S. Il triflusal nella prevenzione degli accidenti cerebrovascolari. G Gerontol 1995;43:563-70.

63. CAPRIE Steering Committee. A randomised, blinded, trial of clopidogrel versus aspirin in patients at risk of ischaemic events (CAPRIE). Lancet 1996;348:1329-39.

64. International Stroke Trial Collaborative Group. The International Stroke Trial (IST): a randomised trial of aspirin, subcutaneous heparin, both, or neither among 19435 patients with acute ischaemic stroke. Lancet 1997;349:1569-81.

65. Matías-Guiu J, Ferro JM, Alvarez-Sabín J, et al. Comparison of triflusal and aspirin for prevention of vascular events in patients after cerebral infarction: the TACIP Study: a randomized, double-blind, multicenter trial. Stroke 2003;34:840-8.

66. Culebras A, Rotta-Escalante R, Vila J, et al. Triflusal vs aspirin for prevention of cerebral infarction: a randomized stroke study. Neurology 2004;62:1073-80.

67. Chairangsarit P, Sithinamsuwan P, Niyasom S, et al. Comparison between aspirin combined with dipyridamole versus aspirin alone within 48 hours after ischemic stroke event for prevention of recurrent stroke and improvement 
of neurological function: a preliminary study. J Med Assoc Thai 2005;88 Suppl 3:S148-54.

68. Lee YS, Bae HJ, Kang DW, et al. Cilostazol in Acute Ischemic Stroke Treatment (CAIST Trial): a randomized double-blind non-inferiority trial. Cerebrovasc Dis 2011;32:65-71.

69. Nakamura T, Tsuruta S, Uchiyama S. Cilostazol combined with aspirin prevents early neurological deterioration in patients with acute ischemic stroke: a pilot study. J Neurol Sci 2012;313:22-6.

70. Benavente OR, Hart RG, McClure LA, et al. Effects of clopidogrel added to aspirin in patients with recent lacunar stroke. N Engl J Med 2012;367:817-25.

71. Yi X, Lin J, Wang C, et al. A comparative study of dual versus monoantiplatelet therapy in patients with acute large-artery atherosclerosis stroke. J Stroke Cerebrovasc Dis 2014;23:1975-81.

72. He F, Xia C, Zhang JH, et al. Clopidogrel plus aspirin versus aspirin alone for preventing early neurological deterioration in patients with acute ischemic stroke. J Clin Neurosci 2015;22:83-6.

73. ESPRIT Study Group; Halkes PH, van Gijn J, et al. Aspirin plus dipyridamole versus aspirin alone after cerebral ischaemia of arterial origin (ESPRIT): randomised controlled trial. Lancet 2006;367:1665-73.

74. Yi X, Chi W, Wang C, et al. Low-molecular-weight heparin or dual antiplatelet therapy is more effective than aspirin alone in preventing early neurological deterioration and improving the 6-month outcome in ischemic stroke patients. J Clin Neurol 2015;11:57-65.

75. Aoki J, Iguchi Y, Urabe T, et al. Acute Aspirin Plus Cilostazol Dual Therapy for Noncardioembolic Stroke Patients Within 48 Hours of Symptom Onset. J Am Heart Assoc 2019;8:e012652.

76. Fields WS, Lemak NA, Frankowski RF, et al. Controlled trial of aspirin in cerebral ischemia. Stroke 1977;8:301-14.

77. Canadian Cooperative Study Group. A randomized trial of aspirin and sulfinpyrazone in threatened stroke. N Engl J Med 1978;299:53-9.

78. Reuther R, Dorndorf W, Loew D. Behandlung transitorisch-ischämischer Attacken mit Azetylsalizylsäure. Munch Med Wochenschr 1980;122:795-8.

79. Bousser MG, Eschwege E, Haguenau M. 'AICLA' controlled trial of aspirin and dipyridamole in the secondary prevention of athero-thrombotic cerebral ischemia. Stroke 1983;14:5-14.

80. Sorensen PS, Pedersen H, Marquardsen J, et al. Acetylsalicylic acid in the prevention of stroke in patients with reversible cerebral ischemic attacks. A Danish cooperative study. Stroke 1983;14:15-22.

81. Tohgi H. The effect of ticlopidine on TIA compared with aspirin: a double-blind, twelve-month follow-up study. Agents Actions Suppl 1984;15:279-82.

82. American-Canadian Co-Operative Study Group. Persantine Aspirin Trial in cerebral ischemia. Part II: Endpoint results. Stroke 1985;16:406-15.

83. Swedish Cooperative Study. High-dose acetylsalicylic acid after cerebral infarction. Stroke 1987;18:325-34.

84. Hass WK, Easton JD, Adams HP Jr, et al. A randomized trial comparing ticlopidine hydrochloride with aspirin for the prevention of stroke in high-risk patients. Ticlopidine Aspirin Stroke Study Group. N Engl J Med 1989;321:501-7.

85. Kaye J, Capildeo R. A trial to evaluate the relative roles of dipyridamole and aspirin in the prevention of deep vein thrombosis in stroke patients. Bracknell: Boehringer Ingelheim (internal report), 1990.

86. Gorelick PB, Richardson D, Kelly M, et al. Aspirin and ticlopidine for prevention of recurrent stroke in black patients: a randomized trial. JAMA 2003;289:2947-57.

87. Gotoh F, Tohgi H, Hirai S, et al. Cilostazol stroke prevention study: A placebo-controlled double-blind trial for secondary prevention of cerebral infarction. J Stroke Cerebrovasc Dis 2000;9:147-57.

88. Fukuuchi Y, Tohgi H, Okudera T, et al. A randomized, double-blind study comparing the safety and efficacy of clopidogrel versus ticlopidine in Japanese patients with noncardioembolic cerebral infarction. Cerebrovasc Dis 2008;25:40-9.

89. Sacco RL, Diener HC, Yusuf S, et al. Aspirin and extended-release dipyridamole versus clopidogrel for recurrent stroke. N Engl J Med 2008;359:1238-51.

90. Serebruany VL, Malinin AI, Pokov AN, et al. Antiplatelet profiles of the fixed-dose combination of extendedrelease dipyridamole and low-dose aspirin compared with clopidogrel with or without aspirin in patients with type 2 diabetes and a history of transient ischemic attack: a randomized, single-blind, 30-day trial. Clin Ther 2008;30:249-59.

91. Uchiyama S, Fukuuchi Y, Yamaguchi T. The safety and efficacy of clopidogrel versus ticlopidine in Japanese stroke patients: combined results of two Phase III, multicenter, randomized clinical trials. J Neurol 2009;256:888-97.

92. Han SW, Kim YJ, Ahn SH, et al. Effects of Triflusal and Clopidogrel on the Secondary Prevention of Stroke Based on Cytochrome P450 2C19 Genotyping. J Stroke 
2017;19:356-64.

93. Ogawa A, Toyoda K, Kitagawa K, et al. Comparison of prasugrel and clopidogrel in patients with noncardioembolic ischaemic stroke: a phase 3, randomised, non-inferiority trial (PRASTRO-I). Lancet Neurol 2019;18:238-47.

94. Acheson J, Danta G, Hutchinson EC. Controlled trial of dipyridamole in cerebral vascular disease. Br Med J 1969;1:614-5.

95. Turpie A, Dobkin B, McKenna R. A trial of ticlopidine, an antiplatelet agent, for acute cerebral infarction. Guildford: Sanofi Winthrop (Sanofi internal report 1983001 6188) $1983 ; 2007$.

96. Ross Russell RW. The effect of ticlopidine in patients with amaurosis fugax. Guildford: Sanofi Winthrop 1985.

97. Gent M, Blakely JA, Easton JD, et al. The Canadian American Ticlopidine Study (CATS) in thromboembolic stroke. Lancet 1989;1:1215-20.

98. Ito E, Takahashi A, Yamamoto H, et al. Ticlopidine alone versus ticlopidine plus aspirin for preventing recurrent stroke. Intern Med 2003;42:793-9.

99. Kwon SU, Hong KS, Kang DW, et al. Efficacy and safety of combination antiplatelet therapies in patients with symptomatic intracranial atherosclerotic stenosis. Stroke 2011;42:2883-90.

100. Diener HC, Bogousslavsky J, Brass LM, et al. Aspirin and clopidogrel compared with clopidogrel alone after recent ischaemic stroke or transient ischaemic attack in high-risk patients (MATCH): randomised, double-blind, placebo-

Cite this article as: Jung SJ, Kim BJ, Kim CK, Shim SR, Jung JM. Antiplatelet regimens after ischemic stroke or transient ischemic attack: a systematic review and updated network meta-analysis. Ann Transl Med 2022;10(5):245. doi: 10.21037/atm-21-3748 controlled trial. Lancet 2004;364:331-7.

101. Wang Y, Chen W, Lin Y, et al. Ticagrelor plus aspirin versus clopidogrel plus aspirin for platelet reactivity in patients with minor stroke or transient ischaemic attack: open label, blinded endpoint, randomised controlled phase II trial. BMJ 2019;365:12211.

102. Pince J. Thromboses veineuses des membres inferieures et embolies pulmonaires au cours des accidents vasculaires cerebraux. A propos d'un essai comparatif de traitment preventif. MD thesis Toulouse: Université Paul Sabatier 1981.

103.ESPS Group. European Stroke Prevention Study. Stroke 1990;21:1122-30.

104. Goto S. Cilostazol: potential mechanism of action for antithrombotic effects accompanied by a low rate of bleeding. Atheroscler Suppl 2005;6:3-11.

105.Levine GN, Jeong YH, Goto S, et al. Expert consensus document: World Heart Federation expert consensus statement on antiplatelet therapy in East Asian patients with ACS or undergoing PCI. Nat Rev Cardiol 2014;11:597-606.

106. Gorelick PB, Wong KS, Bae HJ, et al. Large artery intracranial occlusive disease: a large worldwide burden but a relatively neglected frontier. Stroke 2008;39:2396-9.

107. Toyoda K, Uchiyama S, Yamaguchi T, et al. Dual antiplatelet therapy using cilostazol for secondary prevention in patients with high-risk ischaemic stroke in Japan: a multicentre, open-label, randomised controlled trial. Lancet Neurol 2019;18:539-48. 
Table S1 Search strategy

\begin{tabular}{|c|c|c|c|c|c|c|}
\hline Search No. & PubMed & Results & Embase & Results & Cochrane Trials & Results \\
\hline \#3 & $\begin{array}{l}\text { Ischemic Attack, Transient[mh] OR } \\
\text { transient ischemic attack*[tw] OR transient } \\
\text { ischaemic attack*[tw] OR transient } \\
\text { brain ischemia*[tw] OR transient brain } \\
\text { ischaemia*[tw] OR transient cerebral } \\
\text { ischemia*[tw] OR transient cerebral } \\
\text { ischaemia*[tw] OR (brain[tw] AND (TIA[tw] } \\
\text { OR TIAs[tw])) }\end{array}$ & 30,088 & $\begin{array}{l}\text { ('transient ischemic attack'/ } \\
\text { exp OR (transient NEAR/5 } \\
\text { isch*mic NEAR/5 attack*):ti,ab } \\
\text { OR (transient NEAR/5 (brain OR } \\
\text { cerebral) NEAR/5 isch*mia*):ti,ab } \\
\text { OR (brain NEAR/5 (TIA OR } \\
\text { TIAs)):ti,ab) }\end{array}$ & 47,458 & $\begin{array}{l}\text { [mh "Ischemic Attack, } \\
\text { Transient"] OR (transient NEAR/5 } \\
\text { isch*mic NEAR/5 attack*):ti,ab } \\
\text { OR (transient NEAR/5 (brain OR } \\
\text { cerebral) NEAR/5 isch*mia*):ti,ab } \\
\text { OR (brain NEAR/5 (TIA OR } \\
\text { TIAs)):ti,ab }\end{array}$ & 2,660 \\
\hline$\# 6$ & $\begin{array}{l}\text { Aspirin[mh] OR aspirin[tw] OR } \\
\text { acetylsalicylic acid[tw] OR acetyl } \\
\text { salicylic acid[tw] OR acetosalicylic } \\
\text { acid[tw] OR Acylpyrin[tw] OR Colfarit[tw] } \\
\text { OR Ecotrin[tw] OR Endosprin[tw] } \\
\text { OR Magnecyl[tw] OR Micristin[tw] } \\
\text { OR Polopirin[tw] OR Polopiryna[tw] } \\
\text { OR Solupsan[tw] OR Zorprin[tw] OR } \\
\text { Acetysal[tw] OR Aloxiprimum[tw] OR } \\
\text { Dispril[tw] OR Easprin[tw] OR Solprin[tw] }\end{array}$ & 71,184 & $\begin{array}{l}\text { ('acetylsalicylic acid'/de OR } \\
\text { (aspirin OR 'acetylsalicylic } \\
\text { acid' OR 'acetyl salicylic acid' } \\
\text { OR 'acetosalicylic acid' OR } \\
\text { Acylpyrin OR Colfarit OR Ecotrin } \\
\text { OR Endosprin OR Magnecyl } \\
\text { OR Micristin OR Polopirin } \\
\text { OR Polopiryna OR Solupsan } \\
\text { OR Zorprin OR Acetysal OR } \\
\text { Aloxiprimum OR Dispril OR } \\
\text { Easprin OR Solprin):ti,ab) }\end{array}$ & 226,793 & $\begin{array}{l}\text { [mh Aspirin] OR (aspirin OR } \\
\text { "acetylsalicylic acid" OR "acetyl } \\
\text { salicylic acid" OR "acetosalicylic } \\
\text { acid" OR Acylpyrin OR Colfarit } \\
\text { OR Ecotrin OR Endosprin } \\
\text { OR Magnecyl OR Micristin } \\
\text { OR Polopirin OR Polopiryna } \\
\text { OR Solupsan OR Zorprin OR } \\
\text { Acetysal OR Aloxiprimum } \\
\text { OR Dispril OR Easprin OR } \\
\text { Solprin):ti,ab }\end{array}$ & 14,748 \\
\hline
\end{tabular}

Table S1 (contiuned) 
Table S1 (contiuned)

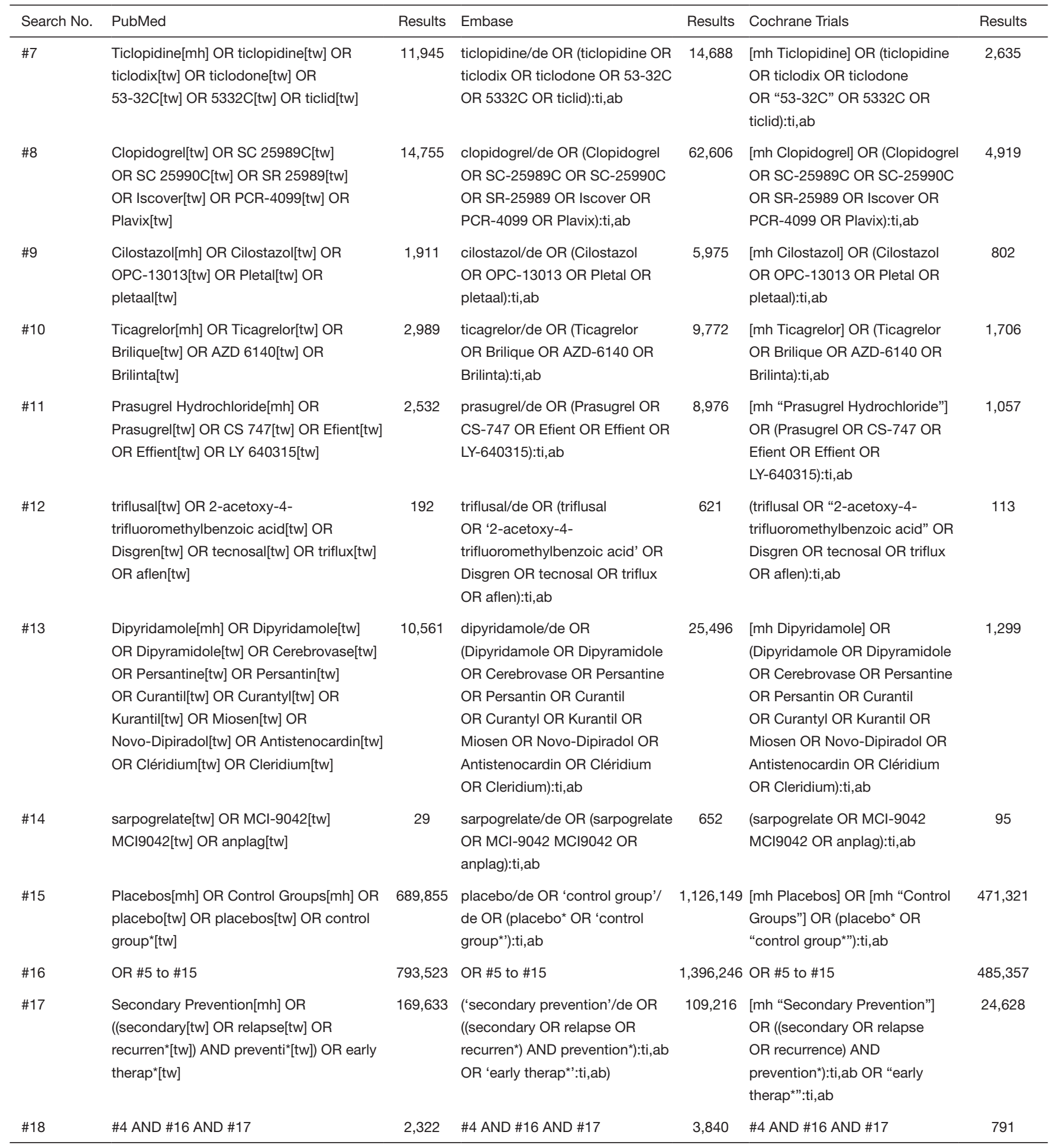

Table S1 (contiuned) 
Table S1 (contiuned)

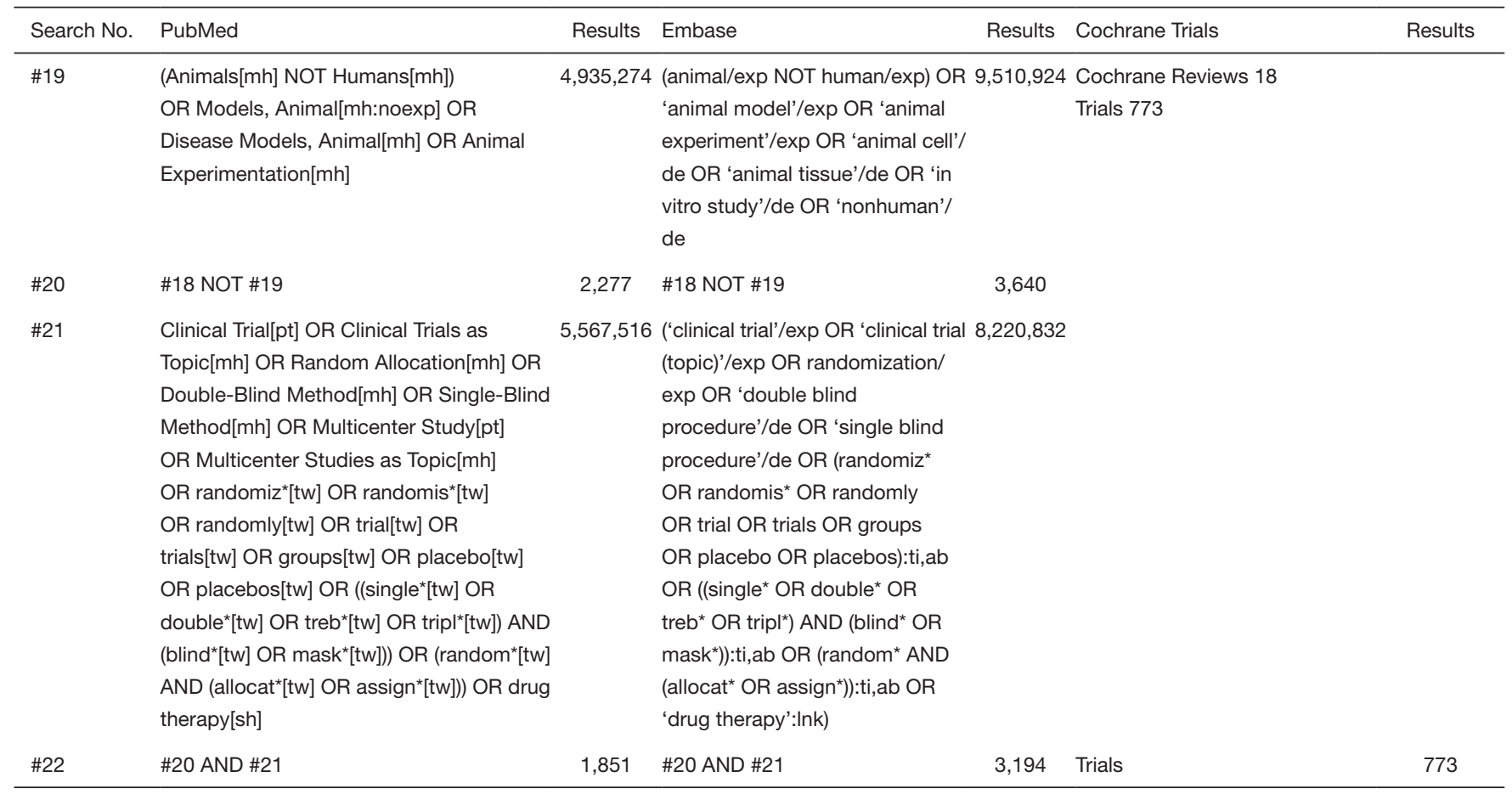

Table S2 Characteristics of the enrolled trials

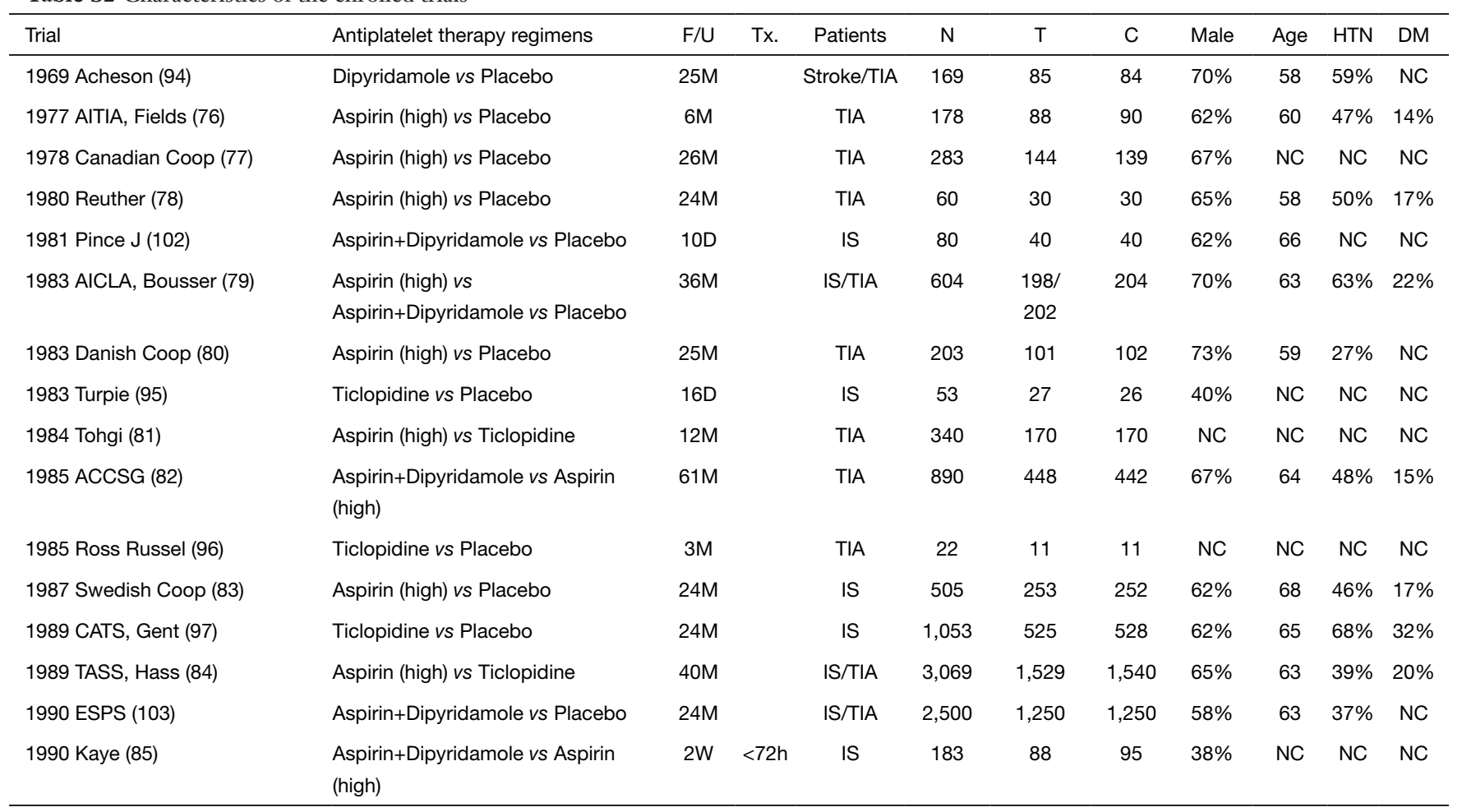

Table S2 (contiuned) 
Table S2 (contiuned)

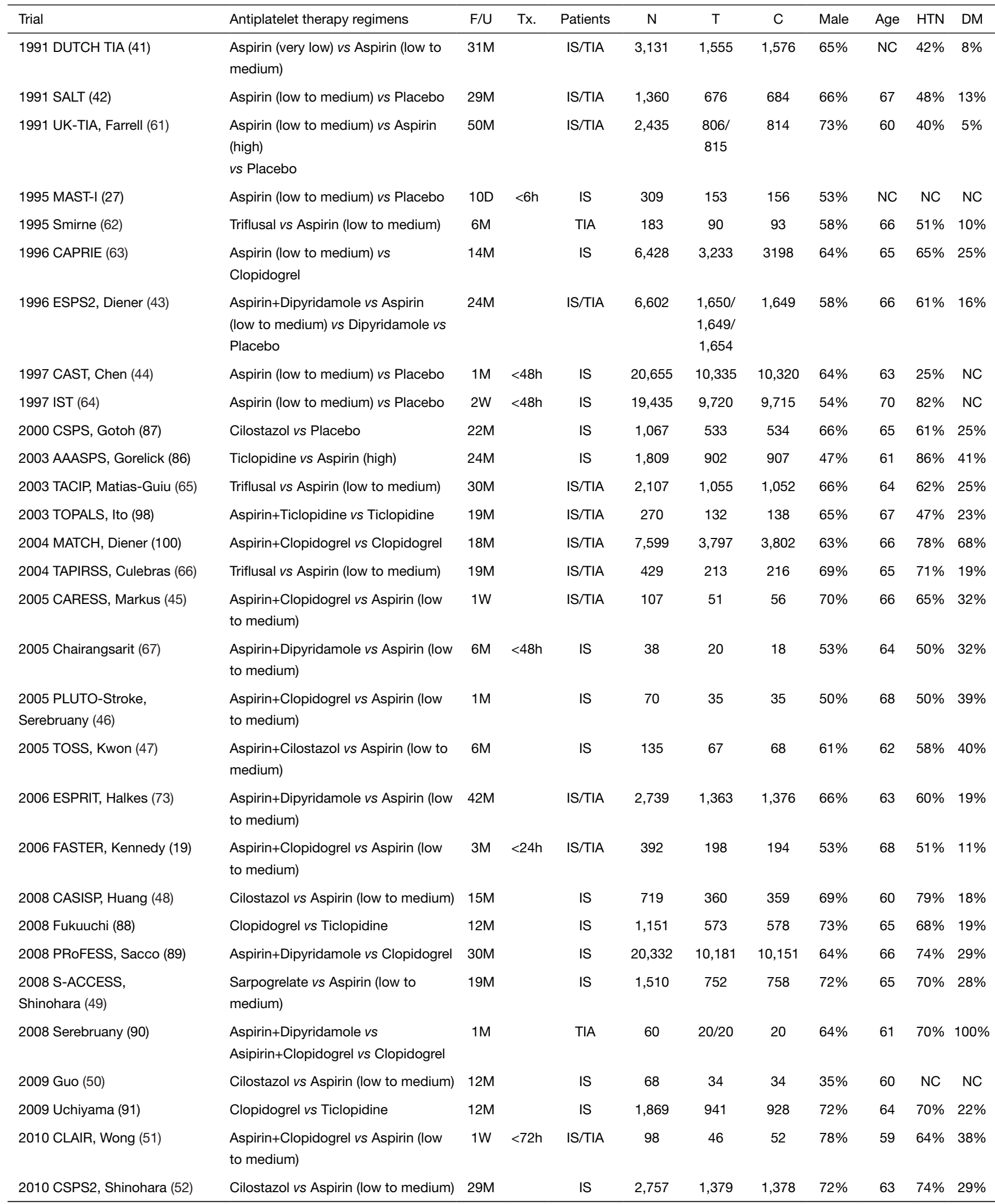

Table S2 (contiuned) 
Table S2 (contiuned)

\begin{tabular}{|c|c|c|c|c|c|c|c|c|c|c|c|}
\hline Trial & Antiplatelet therapy regimens & $F / U$ & Tx. & Patients & $\mathrm{N}$ & $\mathrm{T}$ & C & Male & Age & HTN & DM \\
\hline 2010 EARLY, Dengler (20) & $\begin{array}{l}\text { Aspirin+Dipyridamole vs Aspirin (low } \\
\text { to medium) }\end{array}$ & $1 \mathrm{~W}$ & $<24 \mathrm{~h}$ & IS TIA & 543 & 283 & 260 & $63 \%$ & 69 & $74 \%$ & $24 \%$ \\
\hline 2011 CHARISMA, Hankey (28) & $\begin{array}{l}\text { Aspirin+Clopidogrel vs Aspirin (low } \\
\text { to medium) }\end{array}$ & $25 \mathrm{M}$ & & IS/TIA & 4,320 & 2,157 & 2,163 & $63 \%$ & 65 & $76 \%$ & $29 \%$ \\
\hline 2011 TOSS2, Kwon (99) & $\begin{array}{l}\text { Aspirin+Cilostazol vs } \\
\text { Aspirin+Clopidogrel }\end{array}$ & $7 \mathrm{M}$ & & IS & 457 & 232 & 225 & $52 \%$ & 65 & $72 \%$ & $43 \%$ \\
\hline 2012 Nakamura (69) & $\begin{array}{l}\text { Aspirin+Cilostazol vs Aspirin (low to } \\
\text { medium) }\end{array}$ & $6 \mathrm{M}$ & $<48 \mathrm{~h}$ & IS & 76 & 38 & 38 & $74 \%$ & 66 & $82 \%$ & $35 \%$ \\
\hline 2012 SPS3, Benavente (70) & $\begin{array}{l}\text { Aspirin+Clopidogrel vs Aspirin (low } \\
\text { to medium) }\end{array}$ & $40 \mathrm{M}$ & & IS & 3,020 & 1,517 & 1,503 & $63 \%$ & 63 & $75 \%$ & $37 \%$ \\
\hline 2013 ECLIPse, Han (54) & $\begin{array}{l}\text { Aspirin+Cilostazol vs Aspirin (low to } \\
\text { medium) }\end{array}$ & $3 \mathrm{M}$ & & IS & 203 & 100 & 103 & $75 \%$ & 65 & $57 \%$ & $29 \%$ \\
\hline 2014 Yi (71) & $\begin{array}{l}\text { Aspirin+Clopidogrel vs Aspirin (low } \\
\text { to medium) }\end{array}$ & $1 \mathrm{M}$ & $<48 \mathrm{~h}$ & IS & 574 & 286 & 288 & $55 \%$ & 69 & $73 \%$ & $38 \%$ \\
\hline 2015 CATHARSIS, Uchiyama (55) & $\begin{array}{l}\text { Aspirin+Cilostazol vs Aspirin (low to } \\
\text { medium) }\end{array}$ & $24 \mathrm{M}$ & & IS & 163 & 83 & 80 & $66 \%$ & 68 & $77 \%$ & $37 \%$ \\
\hline $2015 \mathrm{He}(72)$ & $\begin{array}{l}\text { Aspirin+Clopidogrel vs Aspirin (low } \\
\text { to medium) }\end{array}$ & $2 \mathrm{~W}$ & $<72 \mathrm{~h}$ & IS/TIA & 690 & 343 & 347 & $57 \%$ & 62 & $68 \%$ & $42 \%$ \\
\hline 2015 Yi (74) & $\begin{array}{l}\text { Aspirin+Clopidogrel vs Aspirin (low } \\
\text { to medium) }\end{array}$ & $6 \mathrm{M}$ & $<48 \mathrm{~h}$ & IS & 979 & 490 & 489 & $56 \%$ & 69 & $71 \%$ & $34 \%$ \\
\hline 2018 PICASSO, Kim (59) & Cilostazol vs Aspirin (low to medium) & $23 \mathrm{M}$ & & IS/TIA & 1,534 & 766 & 768 & $62 \%$ & 65 & $89 \%$ & $33 \%$ \\
\hline 2018 POINT, Johnston (22) & $\begin{array}{l}\text { Aspirin+Clopidogrel vs Aspirin (low } \\
\text { to medium) }\end{array}$ & $3 \mathrm{M}$ & $<12 \mathrm{~h}$ & IS/TIA & 4,881 & 2,432 & 2,449 & $56 \%$ & 65 & $70 \%$ & $28 \%$ \\
\hline 2019 ADS, Aoki (75) & $\begin{array}{l}\text { Aspirin+Cilostazol vs Aspirin (low to } \\
\text { medium) }\end{array}$ & $3 \mathrm{M}$ & $<48 \mathrm{~h}$ & IS & 1201 & 600 & 601 & $66 \%$ & 69 & $76 \%$ & $32 \%$ \\
\hline 2019 PRASTRO-I, Ogawa (93) & Prasugrel vs Clopidogrel & $25 \mathrm{M}$ & & IS & 3,747 & 1,885 & 1,862 & $79 \%$ & 62 & $80 \%$ & $33 \%$ \\
\hline 2019 PRINCE, Wang (101) & $\begin{array}{l}\text { Aspirin+Ticagrelor vs } \\
\text { Aspirin+Clopidogrel }\end{array}$ & $3 \mathrm{M}$ & $<24 h$ & IS/TIA & 675 & 336 & 339 & $74 \%$ & 60 & $61 \%$ & $25 \%$ \\
\hline 2020 THALES, Johnston (60) & $\begin{array}{l}\text { Aspirin+Ticagrelor vs Aspirin (low to } \\
\text { medium) }\end{array}$ & $1 \mathrm{M}$ & $<24 h$ & IS/TIA & 11,016 & 5,523 & 5,493 & $62 \%$ & 65 & $78 \%$ & $29 \%$ \\
\hline
\end{tabular}

F/U, follow-up period; Tx., time interval from symptom onset to treatment initiation; N, number of total participants; T, number of treatment groups; C, number of comparator groups; IS, ischemic stroke; TIA, transient ischemic stroke; M, month; D, day; W, week 


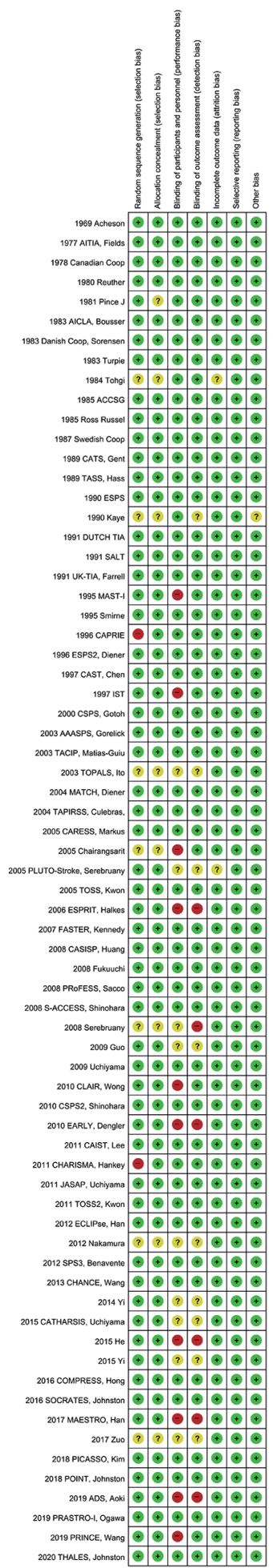

Figure S1 Risk of bias summaries depicted using colors (red: high risk; green: low risk; yellow: unclear). 


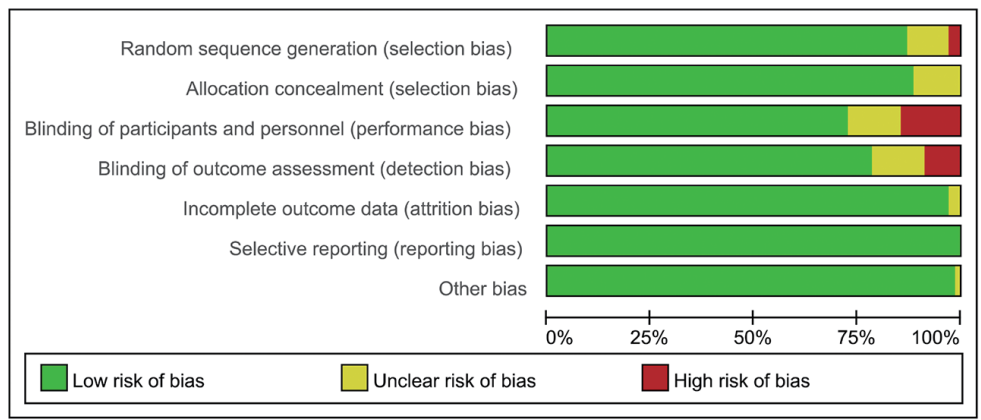

Figure S2 Graph of the total risk of bias of the entire network meta-analysis 
Table S3 League table of antiplatelet regimens with the relative risks and $95 \%$ credible intervals for recurrent stroke

\begin{tabular}{|c|c|c|c|c|c|c|c|c|c|c|c|c|c|c|c|c|}
\hline Antiplatelet Regimens & A_2 & A_3 & A_C & A_Ci & A_D & $\mathrm{A}_{-} \mathrm{T}$ & A_Ti & Cilostazol & Clopidogrel & Dipyridamole & Placebo & Prasugrel & Sarpogrelate & Ticagrelor & Ticlopidine & Triflusal \\
\hline A_2 & & $1.05(0.90,1.26)$ & $1.36(1.22,1.54)$ & $1.32(0.79,2.34)$ & $1.24(1.09,1.39)$ & $0.87(0.29,2.22)$ & $1.29(1.06,1.63)$ & $1.52(1.24,1.85)$ & $1.27(1.08,1.48)$ & $0.95(0.76,1.18)$ & $0.85(0.76,0.93)$ & $1.28(0.86,1.9)$ & $0.88(0.62,1.24)$ & $1.15(0.92,1.42)$ & $1.18(0.96,1.46)$ & $1.01(0.79,1.29)$ \\
\hline A_3 & $0.94(0.79,1.1)$ & & $1.29(1.05,1.58)$ & $1.26(0.71,2.29)$ & $1.17(0.97,1.40)$ & $0.83(0.29,2.08)$ & $1.22(0.95,1.63)$ & $1.43(1.10,1.87)$ & $1.20(0.96,1.49)$ & $0.90(0.68,1.15)$ & $0.80(0.67,0.92)$ & $1.21(0.79,1.84)$ & $0.83(0.57,1.22)$ & $1.08(0.82,1.42)$ & $1.12(0.94,1.32)$ & $0.96(0.70,1.27)$ \\
\hline A_C & $0.73(0.64,0.81)$ & $0.77(0.63,0.94)$ & & $0.96(0.57,1.70)$ & $0.91(0.77,1.04)$ & $0.63(0.22,1.62)$ & $0.94(0.76,1.20)$ & $1.11(0.88,1.38)$ & $0.93(0.78,1.08)$ & $0.69(0.53,0.88)$ & $0.62(0.53,0.71)$ & $0.93(0.62,1.39)$ & $0.64(0.44,0.91)$ & $0.84(0.65,1.06)$ & $0.86(0.68,1.09)$ & $0.74(0.56,0.95)$ \\
\hline A_Ci & $0.75(0.42,1.25)$ & $0.79(0.43,1.39)$ & $1.03(0.58,1.72)$ & & $0.93(0.52,1.59)$ & $0.64(0.19,1.86)$ & $0.97(0.54,1.73)$ & $1.14(0.63,1.98)$ & $0.95(0.53,1.62)$ & $0.71(0.39,1.25)$ & $0.64(0.36,1.07)$ & $0.96(0.49,1.84)$ & $0.66(0.34,1.21)$ & $0.87(0.47,1.51)$ & $0.88(0.48,1.58)$ & $0.75(0.41,1.34)$ \\
\hline A_D & $0.80(0.71,0.91)$ & $0.85(0.71,1.02)$ & $1.09(0.95,1.29)$ & $1.06(0.62,1.88)$ & & $0.70(0.23,1.79)$ & $1.04(0.83,1.36)$ & $1.22(0.97,1.53)$ & $1.02(0.88,1.19)$ & $0.76(0.60,0.97)$ & $0.68(0.59,0.78)$ & $1.03(0.69,1.52)$ & $0.70(0.49,1.02)$ & $0.92(0.72,1.18)$ & $0.95(0.76,1.20)$ & $0.81(0.62,1.07)$ \\
\hline A_T & $1.13(0.44,3.42)$ & $1.20(0.47,3.42)$ & $1.56(0.61,4.48)$ & $1.54(0.53,5.05)$ & $1.41(0.55,4.22)$ & & $1.49(0.58,4.26)$ & $1.73(0.67,5.47)$ & $1.45(0.56,4.34)$ & $1.08(0.42,3.22)$ & $0.96(0.38,2.88)$ & $1.46(0.54,4.71)$ & $1.00(0.38,2.97)$ & $1.30(0.50,4.01)$ & $1.34(0.55,4.01)$ & $1.15(0.44,3.63)$ \\
\hline A_Ti & $0.77(0.61,0.93)$ & $0.81(0.61,1.04)$ & $1.05(0.82,1.30)$ & $1.02(0.57,1.84)$ & $0.96(0.73,1.19)$ & $0.67(0.23,1.72)$ & & $1.16(0.86,1.56)$ & $0.98(0.73,1.25)$ & $0.73(0.52,0.97)$ & $0.65(0.50,0.81)$ & $0.98(0.62,1.52)$ & $0.67(0.44,1.00)$ & $0.88(0.64,1.17)$ & $0.91(0.66,1.20)$ & $0.78(0.55,1.06)$ \\
\hline Cilostazol & $0.65(0.53,0.80)$ & $0.69(0.53,0.90)$ & $0.89(0.72,1.12)$ & $0.87(0.50,1.57)$ & $0.81(0.64,1.02)$ & $0.57(0.18,1.47)$ & $0.85(0.64,1.15)$ & & $0.83(0.64,1.07)$ & $0.62(0.46,0.84)$ & $0.55(0.45,0.69)$ & $0.84(0.54,1.31)$ & $0.58(0.38,0.85)$ & $0.75(0.56,1.00)$ & $0.77(0.58,1.04)$ & $0.66(0.48,0.91)$ \\
\hline Dipyridamole & $1.04(0.84,1.31)$ & $1.10(0.86,1.45)$ & $1.43(1.12,1.86)$ & $1.39(0.79,2.53)$ & $1.30(1.02,1.65)$ & $0.92(0.31,2.37)$ & $1.36(1.02,1.89)$ & $1.59(1.18,2.16)$ & $1.33(1.02,1.74)$ & & $0.89(0.71,1.12)$ & $1.34(0.85,2.12)$ & $0.92(0.62,1.39)$ & $1.20(0.89,1.64)$ & $1.24(0.93,1.68)$ & $1.06(0.77,1.48)$ \\
\hline Placebo & $1.17(1.06,1.29)$ & $1.23(1.07,1.47)$ & $1.60(1.40,1.87)$ & $1.56(0.92,2.77)$ & $1.46(1.27,1.66)$ & $1.03(0.34,2.60)$ & $1.52(1.22,1.96)$ & $1.78(1.44,2.21)$ & $1.49(1.25,1.78)$ & $1.12(0.88,1.39)$ & & $1.50(1.00,2.25)$ & $1.03(0.72,1.48)$ & $1.35(1.07,1.70)$ & $1.39(1.14,1.71)$ & $1.19(0.92,1.55)$ \\
\hline Prasugrel & $0.77(0.52,1.16)$ & $0.82(0.54,1.25)$ & $1.06(0.71,1.60)$ & $1.03(0.54,2.00)$ & $0.96(0.65,1.43)$ & $0.68(0.21,1.83)$ & $1.01(0.65,1.60)$ & $1.19(0.76,1.85)$ & $0.99(0.69,1.43)$ & $0.74(0.47,1.16)$ & $0.66(0.44,0.99)$ & & $0.68(0.40,1.17)$ & $0.89(0.57,1.40)$ & $0.92(0.59,1.41)$ & $0.78(0.50,1.24)$ \\
\hline Sarpogrelate & $1.13(0.80,1.60)$ & $1.19(0.81,1.75)$ & $1.54(1.08,2.24)$ & $1.50(0.82,2.88)$ & $1.41(0.97,2.01)$ & $0.99(0.33,2.63)$ & $1.47(0.99,2.23)$ & $1.71(1.16,2.57)$ & $1.44(0.98,2.10)$ & $1.08(0.71,1.61)$ & $0.96(0.67,1.38)$ & $1.45(0.85,2.46)$ & & $1.30(0.86,1.95)$ & $1.34(0.89,1.99)$ & $1.14(0.76,1.74)$ \\
\hline Ticagrelor & $0.86(0.70,1.07)$ & $0.91(0.70,1.20)$ & $1.18(0.94,1.53)$ & $1.14(0.65,2.11)$ & $1.07(0.84,1.37)$ & $0.76(0.24,1.97)$ & $1.12(0.84,1.56)$ & $1.31(0.99,1.77)$ & $1.10(0.84,1.44)$ & $0.82(0.60,1.12)$ & $0.73(0.58,0.93)$ & $1.11(0.70,1.74)$ & $0.76(0.51,1.15)$ & & $1.02(0.77,1.38)$ & $0.87(0.64,1.21)$ \\
\hline Ticlopidine & $3(0.68,1.03)$ & $0.88(0.75,1.05)$ & $1.15(0.91,1.46)$ & $1.12(0.63,2.05)$ & $1.04(0.83,1.30)$ & $0.74(0.24,1.80)$ & $1.09(0.82,1.50)$ & $1.28(0.96,1.71)$ & $1.07(0.83,1.36)$ & $0.80(0.59,1.07)$ & $0.71(0.58,0.87)$ & $1.08(0.70,1.67)$ & $0.74(0.50,1.11)$ & $0.97(0.72,1.29)$ & & $0.85(0.61,1.16)$ \\
\hline Triflusal & $0.98(0.77,1.25)$ & $1.03(0.78,1.41)$ & $1.34(1.04,1.76)$ & $1.32(0.74,2.41)$ & $1.22(0.93,1.59)$ & $0.86(0.27,2.22)$ & $1.27(0.94,1.79)$ & $1.50(1.09,2.04)$ & $1.25(0.95,1.65)$ & $0.93(0.67,1.29)$ & $0.83(0.64,1.08)$ & $1.26(0.80,1.98)$ & $0.87(0.57,1.30)$ & $1.13(0.82,1.55)$ & $1.17(0.85,1.62)$ & \\
\hline
\end{tabular}

A_z, low to medium dose aspirin; A_3, high dose aspirin; A_C, aspirin plus clopidogrel; A_Ci, aspirin plus cilostazol; A_D, aspirin plus dipyridamole; A_T, aspirin plus ticlopidine; A_Ti, aspirin plus ticagrelor

Table S4 League table of antiplatelet regimens with the relative risks and $95 \%$ credible intervals for recurrent ischemic stroke

\begin{tabular}{|c|c|c|c|c|c|c|c|c|c|c|c|c|c|c|c|c|}
\hline Antiplatelet Regimens & A_2 & A_3 & A_C & A_Ci & A_D & $A_{-} T$ & A_Ti & Cilostazol & Clopidogrel & Dipyridamole & Placebo & Prasugrel & Sarpogrelate & Ticagrelor & Ticlopidine & Triflusal \\
\hline A_2 & & $0.96(0.81,1.14)$ & $1.37(1.25,1.53)$ & $1.15(0.64,2.19)$ & $1.17(1.02,1.32)$ & $1.15(0.39,3.06)$ & $1.31(1.11,1.60)$ & $1.34(1.08,1.62)$ & $4.57(1.04,1.31)$ & $0.68(0.35,1.29)$ & $0.76(0.69,0.85)$ & $1.06(0.75,1.54)$ & $0.78(0.55,1.12)$ & $1.14(0.95,1.36)$ & $1.19(0.95,1.56)$ & $0.94(0.73,1.18)$ \\
\hline A_3 & $1.04(0.87,1.23)$ & & $1.43(1.18,1.74)$ & $1.22(0.67,2.33)$ & $1.21(1.00,1.47)$ & $1.18(0.41,3.12)$ & $1.37(1.06,1.77)$ & $1.37(1.06,1.82)$ & $1.21(1.00,1.47)$ & $0.70(0.35,1.36)$ & $0.80(0.65,0.95)$ & $1.11(0.76,1.67)$ & $0.81(0.55,1.21)$ & $1.19(0.92,1.52)$ & $1.24(1.02,1.52)$ & $0.97(0.72,1.30)$ \\
\hline A_C & $0.72(0.64,0.79)$ & $0.69(0.57,0.84)$ & & $0.84(0.47,1.59)$ & $0.85(0.72,0.98)$ & $0.83(0.28,2.23)$ & $0.95(0.79,1.17)$ & $0.97(0.76,1.21)$ & $0.84(0.73,0.95)$ & $0.49(0.25,0.93)$ & $0.55(0.47,0.64)$ & $0.77(0.54,1.11)$ & $0.56(0.39,0.82)$ & $0.83(0.66,1.00)$ & $0.86(0.67,1.14)$ & $0.68(0.52,0.87)$ \\
\hline A_Ci & $0.86(0.45,1.55)$ & $0.81(0.42,1.49)$ & $1.18(0.62,2.10)$ & & $1.01(0.53,1.76)$ & $1.01(0.25,2.94)$ & $1.13(0.58,2.05)$ & $1.15(0.59,2.03)$ & $1.00(0.52,1.78)$ & $0.59(0.22,1.28)$ & $0.65(0.34,1.16)$ & $0.92(0.45,1.74)$ & $0.67(0.32,1.32)$ & $0.98(0.50,1.79)$ & $1.01(0.51,1.83)$ & $0.81(0.40,1.45)$ \\
\hline A_D & $0.84(0.75,0.97)$ & $0.82(0.67,0.99)$ & $1.17(1.01,1.38)$ & $0.98(0.56,1.87)$ & & $0.97(0.32,2.59)$ & $1.12(0.91,1.43)$ & $1.13(0.89,1.44)$ & $0.98(0.89,1.13)$ & $0.58(0.30,1.10)$ & $0.65(0.56,0.77)$ & $0.91(0.63,1.32)$ & $0.66(0.46,0.98)$ & $0.97(0.78,1.21)$ & $1.02(0.80,1.34)$ & $0.79(0.61,1.04)$ \\
\hline A_T & $0.86(0.32,2.53)$ & $0.84(0.31,2.43)$ & $1.19(0.44,3.49)$ & $0.98(0.33,3.97)$ & $1.02(0.38,3.03)$ & & $1.11(0.42,3.45)$ & $1.15(0.42,3.49)$ & $1.01(0.38,2.97)$ & $0.59(0.18,2.07)$ & $0.65(0.25,1.96)$ & $0.90(0.32,2.94)$ & $0.66(0.23,2.08)$ & $0.99(0.37,2.94)$ & $1.03(0.41,3.03)$ & $0.80(0.29,2.41)$ \\
\hline A_Ti & $0.76(0.62,0.89)$ & $0.72(0.56,0.93)$ & $1.04(0.85,1.26)$ & $0.87(0.48,1.71)$ & $0.89(0.69,1.09)$ & $0.89(0.28,2.34)$ & & $1.01(0.76,1.31)$ & $0.88(0.70,1.07)$ & $0.51(0.26,1.00)$ & $0.58(0.46,0.70)$ & $0.81(0.55,1.21)$ & $0.59(0.39,0.88)$ & $0.86(0.65,1.10)$ & $0.90(0.66,1.24)$ & $0.71(0.51,0.94)$ \\
\hline Cilostazol & $0.74(0.61,0.92)$ & $0.72(0.54,0.94)$ & $1.02(0.82,1.30)$ & $0.86(0.49,1.69)$ & $0.87(0.69,1.11)$ & $0.86(0.28,2.32)$ & $0.98(0.76,1.31)$ & & $0.86(0.69,1.10)$ & $0.51(0.26,1.00)$ & $0.57(0.45,0.72)$ & $0.79(0.54,1.21)$ & $0.58(0.39,0.89)$ & $0.85(0.65,1.12)$ & $0.90(0.65,1.24)$ & $0.69(0.51,0.96)$ \\
\hline Dipyridamole & $1.45(0.77,2.80)$ & $1.41(0.73,2.80)$ & $2.00(1.06,3.93)$ & $1.68(0.77,4.39)$ & $1.71(0.90,3.32)$ & $1.69(0.48,5.41)$ & $1.92(0.99,3.81)$ & $1.94(0.99,3.81)$ & $1.69(0.90,3.28)$ & & $1.11(0.60,2.16)$ & $1.54(0.76,3.35)$ & $1.12(0.57,2.51)$ & $1.66(0.85,3.28)$ & $1.73(0.87,3.59)$ & $1.34(0.70,2.77)$ \\
\hline Placebo & $1.30(1.16,1.44)$ & $1.24(1.04,1.52)$ & $1.79(1.56,2.08)$ & $1.51(0.85,2.85)$ & $1.53(1.28,1.76)$ & $1.51(0.50,3.93)$ & $1.71(1.41,2.13)$ & $1.74(1.43,2.17)$ & $1.52(1.30,1.75)$ & $0.89(0.46,1.65)$ & & $1.39(0.97,2.04)$ & $1.02(0.71,1.48)$ & $1.49(1.20,1.81)$ & $1.56(1.23,2.02)$ & $1.23(0.93,1.57)$ \\
\hline Prasugrel & $0.93(0.64,1.32)$ & $0.89(0.59,1.30)$ & $1.29(0.90,1.82)$ & $1.08(0.57,2.19)$ & $1.09(0.75,1.58)$ & $1.10(0.33,3.03)$ & $1.22(0.82,1.81)$ & $1.25(0.82,1.83)$ & $1.08(0.77,1.51)$ & $0.64(0.29,1.31)$ & $0.71(0.48,1.02)$ & & $0.74(0.43,1.17)$ & $1.07(0.70,1.57)$ & $1.12(0.72,1.68)$ & $0.88(0.57,1.28)$ \\
\hline Sarpogrelate & $1.28(0.88,1.80)$ & $1.22(0.82,1.81)$ & $1.75(1.21,2.51)$ & $1.47(0.75,3.06)$ & $1.50(1.01,2.16)$ & $1.49(0.47,4.17)$ & $1.67(1.12,2.53)$ & $1.71(1.11,2.50)$ & $1.48(1.01,2.17)$ & $0.88(0.39,1.72)$ & $0.97(0.67,1.40)$ & $1.34(0.84,2.28)$ & & $1.46(0.96,2.13)$ & $1.52(0.99,2.34)$ & $1.19(0.78,1.79)$ \\
\hline Ticagrelor & $0.87(0.73,1.04)$ & $0.84(0.65,1.07)$ & $1.19(0.99,1.49)$ & $1.01(0.55,1.99)$ & $1.02(0.82,1.26)$ & $1.00(0.33,2.66)$ & $1.15(0.90,1.51)$ & $1.16(0.88,1.52)$ & $1.01(0.82,1.26)$ & $0.60(0.30,1.17)$ & $0.66(0.54,0.82)$ & $0.93(0.63,1.40)$ & $0.68(0.46,1.03)$ & & $1.03(0.78,1.44)$ & $0.82(0.60,1.09)$ \\
\hline Ticlopidine & $0.83(0.63,1.04)$ & $0.80(0.65,0.97)$ & $1.15(0.87,1.47)$ & $0.98(0.54,1.84)$ & $0.97(0.74,1.23)$ & $0.96(0.32,2.38)$ & $1.10(0.80,1.49)$ & $1.10(0.80,1.52)$ & $0.97(0.75,1.22)$ & $0.57(0.27,1.13)$ & $0.64(0.49,0.81)$ & $0.89(0.59,1.37)$ & $0.65(0.42,1.00)$ & $0.96(0.69,1.28)$ & & $0.78(0.54,1.10)$ \\
\hline Triflusal & $1.05(0.84,1.35)$ & $1.02(0.76,1.37)$ & $1.45(1.14,1.90)$ & $1.22(0.68,2.46)$ & $1.25(0.95,1.62)$ & $1.23(0.41,3.38)$ & $1.39(1.06,1.92)$ & $1.42(1.03,1.94)$ & $1.23(0.96,1.61)$ & $0.74(0.36,1.42)$ & $0.81(0.63,1.07)$ & $1.12(0.77,1.74)$ & $0.83(0.55,1.27)$ & $1.21(0.91,1.64)$ & $1.27(0.90,1.82)$ & \\
\hline
\end{tabular}

$\begin{array}{lllllllllllllll}1.05(0.84,1.35) & 1.02(0.76,1.37) & 1.45(1.14,1.90) & 1.22(0.68,2.46) & 1.25(0.95,1.62) & 1.23(0.41 .3 .38) & 1.39(1.06,1.92) & 1.42(1.03,1.94) & 1.23(0.96,1.61) & 0.74(0.36,1.42) & 0.81(0.63,1.07) & 1.12(0.77 .1 .74) & 0.83(0.55,1.27) & 1.21(0.91 .1 .64) & 1.27(0.90,1.82)\end{array}$

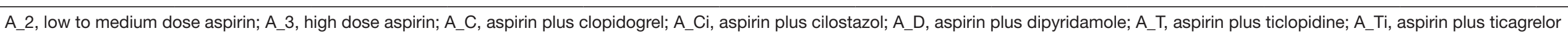


Table S5 League table of antiplatelet regimens with the relative risks and $95 \%$ credible intervals for composite outcomes

\begin{tabular}{|c|c|c|c|c|c|c|c|c|c|c|c|c|c|c|c|c|c|}
\hline intiplatel & A_1 & A_2 & A_3 & A_C & A_Ci & A_D & $A_{-} T$ & $\mathrm{~A}_{-} \mathrm{Ti}$ & Cilostazol & Clopidogrel & Dipyridamole & Placebo & Prasugrel & Sarpogrelate & Ticagrelor & Ticlopidine & Triflusal \\
\hline A_1 & & $0.96(0.79,1.16)$ & $1.07(0.85,1.35)$ & $1.23(0.99,1.51)$ & $1.09(0.69,1.67)$ & $1.16(0.94,1.43)$ & $1.10(0.43,2.80)$ & $1.81(1.03,3.28)$ & $1.32(1.03,1.72)$ & $1.13(0.91,1.40)$ & $0.90(0.69,1.16)$ & $0.84(0.68,1.03)$ & $1.16(0.79,1.72)$ & $0.90(0.63,1.29)$ & $1.08(0.84,1.38)$ & $1.13(0.88,1.45)$ & $0.92(0.70,1.20)$ \\
\hline A_2 & $1.03(0.85,1.25)$ & & $1.10(0.98,1.24)$ & $1.27(1.16,1.39)$ & $1.13(0.76,1.67)$ & $1.20(1.10,1.30)$ & $1.14(0.47,2.85)$ & $1.88(1.12,3.32)$ & $1.37(1.14,1.63)$ & $1.17(1.07,1.28)$ & $0.93(0.78,1.10)$ & $0.87(0.81,0.93)$ & $1.21(0.85,1.68)$ & $0.93(0.69,1.26)$ & $1.12(0.95,1.30)$ & $1.17(1.0$ & $0.95(0.7$ \\
\hline A_3 & $0.93(0.73,1.17)$ & $0.90(0.80,1.01)$ & & $1.14(0.99,1.32)$ & $1.01(0.66,1.54)$ & $1.08(0.95,1.22)$ & $1.03(0.43,2.58)$ & $1.70(0.98,3.00)$ & $1.23(0.98,1.54)$ & $1.05(0.93,1.21)$ & $0.84(0.68,1.03)$ & $0.78(0.70,0.88)$ & $1.08(0.76,1.54)$ & $0.84(0.60,1.17)$ & $1.01(0.83,1.23)$ & $1.05(0.93,1.18)$ & $0.85(0.68,1.06)$ \\
\hline A_C & $0.81(0.65,1.00)$ & $0.78(0.71,0.85)$ & $0.87(0.75,1.00)$ & & $0.89(0.59,1.31)$ & $0.94(0.83,1.05)$ & $0.89(0.36,2.28)$ & $1.47(0.88,2.55)$ & $1.07(0.89,1.30)$ & $0.92(0.82,1.02)$ & $0.73(0.60,0.88)$ & $0.68(0.61,0.76)$ & $0.95(0.66,1.33)$ & $0.73(0.53,1.01)$ & $0.88(0.73,1.04)$ & $0.91(0.77,1.09)$ & $0.74(0.60,0.91)$ \\
\hline A_Ci & $0.91(0.59,1.44)$ & $0.88(0.59,1.31)$ & $0.98(0.64,1.49)$ & $1.12(0.76,1.68)$ & & $1.06(0.71,1.60)$ & $1.01(0.37,2.80)$ & $1.69(0.86,3.12)$ & $1.21(0.78,1.90)$ & $1.04(0.69,1.56)$ & $0.82(0.53,1.27)$ & $0.77(0.52,1.15)$ & $1.07(0.62,1.83)$ & $0.83(0.51,1.40)$ & $0.98(0.65,1.52)$ & $1.04(0.67,1.58)$ & $0.84(0.55,1.34)$ \\
\hline D & $0.86(0.69,1.05)$ & $0.83(0.76,0.90)$ & $0.92(0.81,1.04)$ & $1.05(0.94,1.19)$ & $0.93(0.62,1.40)$ & & $0.94(0.39,2.39)$ & $1.56(0.92,2.77)$ & $1.14(0.93,1.38)$ & $0.97(0.89,1.07)$ & $0.78(0.64,0.93)$ & $0.72(0.66,0.79)$ & $1.00(0.71,1.40)$ & $0.77(0.57,1.06)$ & $0.93(0.77,1.11)$ & $0.97(0.83,1.13)$ & $0.79(0.64,0.96)$ \\
\hline T & $0.90(0.35,2.30)$ & $0.87(0.34,2.10)$ & $0.96(0.38,2.30)$ & $1.11(0.43,2.70)$ & $0.98(0.35,2.65)$ & $1.05(0.41,2.51)$ & & $1.61(0.57,4.71)$ & $1.19(0.48,3.00)$ & $1.02(0.41,2.45)$ & $0.81(0.31,2.07)$ & $0.76(0.30,1.82)$ & $1.05(0.38,2.66)$ & $0.81(0.31,2.10)$ & $0.98(0.38,2.42)$ & $1.02(0.41,2.39)$ & $0.83(0.32,2.08)$ \\
\hline $\mathrm{Ti}$ & $0.55(0.30,0.96)$ & $0.53(0.30,0.89)$ & $0.58(0.33,1.01)$ & $0.67(0.39,1.12)$ & $0.59(0.31,1.15)$ & $0.64(0.36,1.08)$ & $0.62(0.21,1.72)$ & & $0.73(0.41,1.23)$ & $0.62(0.35,1.05)$ & $0.49(0.27,0.84)$ & $0.46(0.26,0.78)$ & $0.64(0.33,1.16)$ & $0.49(0.26,0.93)$ & $0.59(0.32,1.02)$ & $0.62(0.34,1.07)$ & $0.51(0.28,0.87)$ \\
\hline illostazol & $0.75(0.58,0.97)$ & $0.72(0.61,0.87)$ & $0.80(0.64,1.01)$ & $0.92(0.76,1.11)$ & $0.82(0.52,1.26)$ & $0.87(0.72,1.06)$ & $0.83(0.33,2.07)$ & $1.36(0.80,2.41)$ & & $0.85(0.70,1.05)$ & $0.68(0.53,0.87)$ & $0.63(0.53,0.77)$ & $0.88(0.59,1.27)$ & $0.67(0.48,0.96)$ & $0.81(0.64,1.02)$ & $0.85(0.67,1.09)$ & $0.69(0.53,0.89)$ \\
\hline Clopidogrel & $0.88(0.70,1.08)$ & $0.85(0.77,0.92)$ & $0.94(0.82,1.07)$ & $1.08(0.97,1.20)$ & $0.96(0.63,1.43)$ & $1.02(0.93,1.12)$ & $0.97(0.40,2.43)$ & $1.60(0.94,2.82)$ & $1.16(0.94,1.42)$ & & $0.79(0.65,0.95)$ & $0.74(0.66,0.82)$ & $1.02(0.73,1.41)$ & $0.79(0.58,1.09)$ & $0.95(0.79,1.13)$ & $0.99(0.85,1.16)$ & $0.81(0.65,0.99)$ \\
\hline Dipyridamole & $1.10(0.85,1.43)$ & $1.06(0.90,1.26)$ & $1.18(0.96,1.45)$ & $1.35(1.13,1.65)$ & $1.20(0.78,1.85)$ & $1.28(1.07,1.54)$ & $1.22(0.48,3.12)$ & $2.00(1.18,3.59)$ & $1.46(1.14,1.87)$ & $1.25(1.04,1.52)$ & & $0.93(0.78,1.11)$ & $1.28(0.88,1.87)$ & $1.00(0.70,1.41)$ & $1.19(0.95,1.51)$ & $1.25(1.01,1.56)$ & $1.02(0.78,1.30)$ \\
\hline lacebo & $1.18(0.96,1.45)$ & $1.14(1.07,1.22)$ & $1.26(1.13,1.41)$ & $1.45(1.31,1.62)$ & $1.29(0.86,1.91)$ & $1.37(1.25,1.51)$ & $1.30(0.54,3.28)$ & $2.14(1.27,3.78)$ & $1.56(1.29,1.88)$ & $1.33(1.21,1.49)$ & $1.06(0.89,1.26)$ & & $1.38(0.97,1.94)$ & $1.06(0.78,1.45)$ & $1.28(1.07,1.52)$ & $1.34(1.15,1.54)$ & $1.08(0.89,1.32)$ \\
\hline rasugrel & $0.85(0.57,1.26)$ & $0.82(0.59,1.16)$ & $0.92(0.64,1.31)$ & $1.05(0.74,1.50)$ & $0.93(0.54,1.59)$ & $0.99(0.71,1.40)$ & $0.94(0.37,2.56)$ & $1.54(0.85,2.97)$ & $1.13(0.78,1.67)$ & $0.97(0.70,1.35)$ & $0.77(0.53,1.13)$ & $0.72(0.51,1.02)$ & & $0.77(0.48,1.23)$ & $0.92(0.64,1.35)$ & $0.97(0.68,1.39)$ & $0.78(0.53,1.16)$ \\
\hline arpogrel & $1.11(0.77,1.57)$ & $1.06(0.78,1.43)$ & $1.19(0.85,1.65)$ & $1.36(0.98,1.86)$ & $1.19(0.71,1.92)$ & $1.28(0.93,1.74)$ & $1.22(0.47,3.22)$ & $2.02(1.07,3.78)$ & $1.47(1.03,2.06)$ & $1.25(0.91,1.71)$ & $0.99(0.70,1.42)$ & $0.93(0.68,1.26)$ & $1.29(0.81,2.07)$ & & $1.19(0.85,1.67)$ & $0.88,1.77)$ & $1.01(0.70,1.49)$ \\
\hline icagrelor & $2(0.72,1.18)$ & $0.89(0.76,1.04)$ & $0.98(0.81,1.20)$ & $1.13(0.95,1.36)$ & $1.01(0.65,1.53)$ & $1.07(0.90,1.28)$ & $1.01(0.41,2.63)$ & $1.67(0.97,3.03)$ & $1.22(0.97,1.54)$ & $1.04(0.87,1.25)$ & $0.83(0.65,1.04)$ & $0.78(0.65,0.92)$ & $1.08(0.73,1.55)$ & $0.83(0.59,1.17)$ & & $1.04(0.84,1.2$ & $0.85(0.66,1.09)$ \\
\hline iclopidine & $0.88(0.68,1.12)$ & $0.85(0.73,0.98)$ & $0.94(0.84,1.06)$ & $1.08(0.91,1.28)$ & $0.95(0.63,1.47)$ & $1.02(0.87,1.19)$ & $0.97(0.41,2.41)$ & $1.60(0.92,2.85)$ & $1.17(0.91,1.47)$ & $1.00(0.85,1.17)$ & $0.79(0.63,0.98)$ & $0.74(0.64,0.86)$ & $1.02(0.71,1.46)$ & $0.79(0.56,1.12)$ & $0.95(0.77,1.18)$ & & $0.81(0.63,1.03)$ \\
\hline Triflusal & $1.08(0.82,1.42)$ & $1.04(0.87,1.26)$ & $1.16(0.93,1.45)$ & $1.33(1.09,1.65)$ & $1.18(0.74,1.81)$ & $1.25(1.03,1.55)$ & $1.19(0.47,3.09)$ & $1.95(1.13,3.56)$ & $1.43(1.11,1.87)$ & $1.22(1.00,1.51)$ & $0.97(0.76,1.26)$ & $0.91(0.75,1.12)$ & $1.26(0.86,1.87)$ & $0.98(0.67,1.41)$ & $1.17(0.91,1.50)$ & $1.22(0.96,1.57)$ & \\
\hline
\end{tabular}

A_1, very low dose aspirin; A_2, low to medium dose aspirin; A_3 , high dose aspirin; A_C, aspirin plus clopidogrel; A_Ci, aspirin plus cilostazol; A_D, aspirin plus dipyridamole; A_T, aspirin plus ticlopidine; A_Ti, aspirin plus ticagrelor

Table S6 League table of antiplatelet regimens with the relative risks and $95 \%$ credible intervals for major bleeding events

\begin{tabular}{|c|c|c|c|c|c|c|c|c|c|c|c|c|c|c|c|c|}
\hline Antiplatelet Regimens & A_1 & A_2 & A_3 & A_C & A_Ci & A_D & A_T & A_Ti & Cilostazol & Clopidogrel & Dipyridamole & Placebo & Prasugrel & Ticagrelor & Ticlopidine & Triflusal \\
\hline A_1 & & $0.76(0.46,1.24)$ & $0.69(0.36,1.34)$ & $0.43(0.25,0.73)$ & $0.70(0.25,1.84)$ & $0.83(0.48,1.88)$ & $1.46(0.38,4.95)$ & $25(0.11,0.55)$ & $1.95(0.96,4.05)$ & $0.97(0.55,1.71)$ & $1.66(0.80,3.38)$ & $1.21(0.72,2.17)$ & $1.29(0.56,3.03)$ & $0.92(0.45,1.92)$ & $1.49(0.72,3.03)$ & $1.75(0.82,3.74)$ \\
\hline 2 & $1.31(0.80,2.16)$ & & $0.91(0.59,1.34)$ & $0.56(0.45,0.71)$ & $0.91(0.37,2.15)$ & $1.10(0.86,1.39)$ & $1.92(0.55,5.87)$ & $32(0.17,0.61)$ & $2.54(1.58,4.26)$ & $1.27(0.95,1.71)$ & $2.20(1.29,3.70)$ & $1.58(1.32,2.10)$ & $1.70(0.84,3.52)$ & $1.22(0.71,2.09)$ & $1.95(1.16,3.28)$ & $2.29(1.31,4.09)$ \\
\hline 3 & $1.44(0.74,2.74)$ & $1.09(0.74,1.66)$ & & $0.62(0.40,1.00)$ & $0.99(0.38,2.67)$ & $1.22(0.80,1.84)$ & $2.08(0.61,6.42)$ & $36(0.17,0.74)$ & $2.77(1.57,5.47)$ & $1.41(0.90,2.18)$ & $2.39(1.27,4.66)$ & $1.75(1.17,2.80)$ & $1.86(0.88,4.09)$ & $1.34(0.70,2.61)$ & $2.15(1.24,3.63)$ & $2.53(1.30,5.20)$ \\
\hline$c$ & $2.32(1.36,3.93)$ & $1.77(1.40,2.19)$ & $1.60(0.99,2.46)$ & & $1.66(0.65,3.70)$ & $35(1.42,2.59)$ & $3.42(0.96,10.3)$ & $58(0.29,1.11)$ & $4.48(2.67,8.16)$ & $2.25(1.67,3.00)$ & $3.89(2.19,6.75)$ & $2.82(2.09,4.01)$ & $3.00(1.48,6.23)$ & $2.18(1.19,3.89)$ & $3.45(1.99,5.92)$ & $4.05(2.22,7.53)$ \\
\hline $\mathrm{Ci}$ & $1.41(0.54,3.89)$ & $1.08(0.46,2.63)$ & $1.00(0.37,2.61)$ & $0.59(0.26,1.52)$ & & $20(0.51,3.06)$ & $2.02(0.47,8.84)$ & $0.36(0.12,0.95)$ & $2.74(1.06,7.53)$ & $1.36(0.57,3.59)$ & $2.36(0.86,7.02)$ & $1.74(0.72,4.43)$ & $1.82(0.62,6.23)$ & $1.33(0.48,3.63)$ & $2.02(0.79,6.11)$ & $2.44(0.90,7.38)$ \\
\hline D & $1.19(0.69,2.06)$ & $0.90(0.71,1.15)$ & $0.81(0.54,1.23)$ & $0.51(0.38,0.69)$ & $0.83(0.32,1.95)$ & & $1.74(0.49,5.31)$ & $0.30(0.14,0.57)$ & $2.31(1.36,4.13)$ & $1.15(0.89,1.51)$ & $1.97(1.14,3.52)$ & $1.43(1.07,2.08)$ & $1.53(0.78,3.12)$ & $1.10(0.62,2.02)$ & $1.76(1.04,3.06)$ & $2.06(1.15,3.97)$ \\
\hline T & $0.68(0.20,2.59)$ & $0.51(0.17,1.80)$ & $0.47(0.15,1.62)$ & $0.29(0.09,1.03)$ & $0.49(0.11,2.08)$ & $0.57(0.18,2.00)$ & & $0.17(0.04,0.68)$ & $1.33(0.38,5.20)$ & $0.65(0.22,2.31)$ & $1.14(0.32,4.30)$ & $0.88(0.27,3.00)$ & $0.88(0.25,3.63)$ & $0.63(0.18,2.61)$ & $1.02(0.37,3.12)$ & $1.17(0.33,4.61)$ \\
\hline $\mathrm{Ti}$ & $3(1.80,8.75)$ & $3.03(1.62,5.87)$ & $71(1.35,5.69)$ & $1.72(0.89,3.42)$ & $2.77(1.04,7.84)$ & $3.32(1.73,6.68)$ & $5.64(1.46,21.1)$ & & $7.76(3.49,17.4)$ & $3.85(1.96,7.92)$ & $6.55(2.97,15.3)$ & $4.80(2.52,9.97)$ & $5.10(2.07,13.8)$ & $3.70(1.61,8.67)$ & $5.87(2.57,13.7)$ & $6.88(2.9 \mathrm{Y}$ \\
\hline lostaz & $0.51(0.24,1.03)$ & $0.39(0.23,0.63)$ & $0.36(0.18,0.63)$ & $0.22(0.12,0.37)$ & $0.36(0.13,0.94)$ & $0.43(0.24,0.73)$ & $0.74(0.19,2.61)$ & $0.12(0.05,0.28)$ & & $0.50(0.27,0.87)$ & $0.86(0.41,1.70)$ & $0.62(0.37,1.06)$ & $0.67(0.28,1.59)$ & $0.47(0.22,0.97)$ & $0.75(0.36,1.56)$ & $0.88(0.43,1.88)$ \\
\hline opidogrel & $1.03(0.58,1.80)$ & $0.78(0.58,1.04)$ & $0.70(0.45,1.10)$ & $0.44(0.33,0.59)$ & $0.73(0.27,1.72)$ & $0.86(0.65,1.11)$ & $1.51(0.43,4.52)$ & $0.25(0.12,0.50)$ & $1.98(1.14,3.66)$ & & $1.71(0.95,3.09)$ & $1.24(0.90,1.88)$ & $1.33(0.70,2.55)$ & $0.96(0.51,1.79)$ & $1.52(0.91,2.58)$ & $1.78(0.96,3.45)$ \\
\hline yyridamole & $0.60(0.29,1.23)$ & $0.45(0.26,0.76)$ & $0.41(0.21,0.78)$ & $0.25(0.14,0.45)$ & $0.42(0.14,1.15)$ & $0.50(0.28,0.87)$ & $0.87(0.23,3.06)$ & $0.15(0.06,0.33)$ & $1.15(0.58,2.43)$ & $0.58(0.32,1.04)$ & & $0.72(0.42,1.30)$ & $0.77(0.33,1.93)$ & $0.55(0.26,1.19)$ & $0.88(0.42,1.87)$ & $1.04(0.47,2.32)$ \\
\hline acebo & $32(0.45,1.38)$ & $0.62(0.47,0.75)$ & $0.57(0.35,0.85)$ & $0.35(0.24,0.47)$ & $0.57(0.22,1.38)$ & $0.69(0.47,0.92)$ & $1.19(0.33,3.63)$ & $0.20(0.10,0.39)$ & $1.60(0.93,2.69)$ & $0.80(0.53,1.10)$ & $1.37(0.76,2.34)$ & & $1.06(0.50,2.2$ & $0.76(0.41$ & $1.21(0.68$ & $1.43(0.7$ \\
\hline rasugrel & $0.76(0.32,1.76)$ & $0.58(0.28,1.18)$ & $0.53(0.24,1.12)$ & $0.33(0.16,0.67)$ & $0.54(0.16,1.59)$ & $0.65(0.31,1.27)$ & $1.12(0.27,3.93)$ & $0.19(0.07,0.48)$ & $1.47(0.62,3.56)$ & $0.74(0.39,1.41)$ & $1.28(0.51,3.00)$ & $0.94(0.44,1.99)$ & & $0.72(0.28,1.70)$ & $1.12(0.50,2.56)$ & $1.32(0.54,3.38)$ \\
\hline Ticagrelor & $1.08(0.51,2.20)$ & $0.81(0.47,1.40)$ & $0.74(0.38,1.42)$ & $0.45(0.25,0.83)$ & $0.74(0.27,2.07)$ & $0.90(0.49,1.61)$ & $1.58(0.38,5.41)$ & $0.26(0.11,0.61)$ & $2.09(1.02,4.39)$ & $1.03(0.55,1.92)$ & $1.80(0.83,3.81)$ & $1.30(0.74,2.42)$ & $1.38(0.58,3.49)$ & & $1.58(0.73,3.38)$ & $1.87(0.87,4.17)$ \\
\hline lopidine & $6(0.32,1.37)$ & $0.51(0.30,0.85)$ & $6(0.27,0.80)$ & $0.28(0.16,0.50)$ & $0.49(0.16,1.25)$ & $0.56(0.32,0.95)$ & $0.97(0.31,2.67)$ & $0.17(0.07,0.38)$ & $1.32(0.63,2.71)$ & $0.65(0.38,1.09)$ & $1.13(0.53,2.32)$ & $0.82(0.48,1.45)$ & $0.89(0.38,1.98)$ & $0.63(0.29,1.35)$ & & $1.18(0.53,2.51)$ \\
\hline flusal & $0.56(0.26,1.21)$ & $0.43(0.24,0.76)$ & $39(0.19,0.76)$ & $0.24(0.13,0.44)$ & $0.40(0.13,1.09)$ & $0.48(0.25,0.86)$ & $0.84(0.21,2.94)$ & $0.14(0.05,0.34)$ & $1.12(0.53,2.31)$ & $0.56(0.28,1.03)$ & $0.95(0.42,2.08)$ & $0.69(0.38,1.28)$ & $0.75(0.29,1.84)$ & $0.53(0.23,1.14)$ & $0.84(0.39,1.87)$ & \\
\hline
\end{tabular}

A_1, very low dose aspirin; A_2, low to medium dose aspirin; A_3, high dose aspirin; A_C, aspirin plus clopidogrel; A_Ci, aspirin plus cilostazol; A_D, aspirin plus dipyridamole; A_T, aspirin plus ticlopidine; A_Ti, aspirin plus ticagrelor 
Table S7 League table of antiplatelet regimens with the relative risks and 95\% credible intervals for all bleeding events

\begin{tabular}{|c|c|c|c|c|c|c|c|c|c|c|c|c|c|c|c|c|}
\hline Antiplatelet Regimens & A_1 & A_2 & A_3 & A_C & A_Ci & A_D & $A_{-} T \mathrm{~T}$ & Cilostazol & Clopidogrel & Dipyridamole & Placebo & Prasugrel & Sarpogrelate & Ticagrelor & Ticlopidine & Triflusal \\
\hline A_1 & & $0.65(0.37,1.15)$ & $0.47(0.22,0.90)$ & $0.33(0.18,0.62)$ & $0.42(0.12,1.50)$ & $0.64(0.33,1.15)$ & $0.21(0.09,0.50)$ & $1.02(0.55,1.97)$ & $0.79(0.41,1.58)$ & $1.11(0.51,2.31)$ & $0.97(0.51,1.82)$ & $0.77(0.32,1.83)$ & $0.96(0.44,2.09)$ & $0.50(0.23,1.10)$ & $0.55(0.25,1.16)$ & $1.09(0.57,2.26)$ \\
\hline A_2 & $1.52(0.86,2.65)$ & & $0.72(0.47,1.04)$ & $0.51(0.40,0.65)$ & $0.64(0.21,2.02)$ & $0.98(0.72,1.23)$ & $0.32(0.17,0.61)$ & $1.56(1.17,2.12)$ & $1.21(0.84,1.77)$ & $1.69(0.99,2.77)$ & $1.48(1.09,1.99)$ & $1.17(0.60,2.28)$ & $1.46(0.84,2.52)$ & $0.77(0.43,1.34)$ & $0.84(0.50,1.38)$ & $1.67(1.14,2.59)$ \\
\hline A_3 & $2.09(1.10,4.34)$ & $1.37(0.96,2.10)$ & & $0.71(0.46,1.15)$ & $0.89(0.28,2.97)$ & $1.34(0.90,2.02)$ & $0.45(0.22,0.97)$ & $2.16(1.39,3.63)$ & $1.67(1.03,2.91)$ & $2.34(1.29,4.34)$ & $2.05(1.40,3.09)$ & $1.61(0.79,3.56)$ & $2.01(1.06,4.09)$ & $1.06(0.55,2.18)$ & $1.16(0.79,1.78)$ & $2.31(1.39,4.30)$ \\
\hline A_C & $2.94(1.60,5.41)$ & $1.92(1.52,2.44)$ & $1.39(0.86,2.14)$ & & $1.24(0.41,4.01)$ & $1.89(1.31,2.56)$ & $0.63(0.35,1.13)$ & $3.03(2.10,4.43)$ & $2.33(1.61,3.45)$ & $3.25(1.84,5.64)$ & $2.85(1.95,4.17)$ & $2.27(1.17,4.43)$ & $2.82(1.55,5.10)$ & $1.48(0.81,2.71)$ & $1.63(0.92,2.80)$ & $3.22(2.12,5.25)$ \\
\hline A_Ci & $2.34(0.66,8.00)$ & $1.55(0.49,4.57)$ & $1.11(0.33,3.52)$ & $0.80(0.24,2.43)$ & & $1.50(0.46,4.57)$ & $0.51(0.13,1.77)$ & $2.43(0.76,7.53)$ & $1.88(0.56,5.98)$ & $2.61(0.75,8.75)$ & $2.29(0.70,7.17)$ & $1.81(0.49,6.55)$ & $2.27(0.63,7.61)$ & $1.19(0.33,4.05)$ & $1.30(0.38,4.34)$ & $2.62(0.77,8.33)$ \\
\hline A_D & $1.54(0.86,2.97)$ & $1.01(0.80,1.37)$ & $0.74(0.49,1.10)$ & $0.52(0.38,0.75)$ & $0.66(0.21,2.13)$ & & $0.33(0.17,0.67)$ & $1.59(1.12,2.45)$ & $1.23(0.87,1.87)$ & $1.72(1.02,2.94)$ & $1.51(1.08,2.20)$ & $1.19(0.63,2.41)$ & $1.49(0.83,2.82)$ & $0.78(0.43,1.49)$ & $0.86(0.51,1.47)$ & $1.70(1.12,2.88)$ \\
\hline A_Ti & $4.61(1.99,10.5)$ & $3.03(1.62,5.69)$ & $2.20(1.02,4.48)$ & $1.57(0.87,2.82)$ & $1.96(0.56,7.17)$ & $2.97(1.47,5.64)$ & & $4.75(2.40,9.58)$ & $3.70(1.86,7.53)$ & $5.15(2.25,11.3)$ & $4.52(2.24,8.93)$ & $3.56(1.47,8.75)$ & $4.48(1.94,10.3)$ & $2.33(1.01,5.52)$ & $2.56(1.13,5.69)$ & $5.10(2.5,11.02)$ \\
\hline Cilostazol & $0.97(0.50,1.80)$ & $0.63(0.46,0.84)$ & $0.46(0.27,0.71)$ & $0.32(0.22,0.47)$ & $0.41(0.13,1.31)$ & $0.62(0.40,0.89)$ & $0.21(0.10,0.41)$ & & $0.77(0.48,1.23)$ & $1.07(0.58,1.88)$ & $0.94(0.63,1.38)$ & $0.74(0.36,1.53)$ & $0.93(0.49,1.72)$ & $0.49(0.25,0.91)$ & $0.53(0.29,0.92)$ & $1.06(0.65,1.78)$ \\
\hline Dipyridamole & $0.89(0.43,1.93)$ & $0.58(0.36,1.00)$ & $0.42(0.22,0.77)$ & $0.30(0.17,0.54)$ & $0.38(0.11,1.33)$ & $0.57(0.33,0.97)$ & $0.19(0.08,0.44)$ & $0.92(0.52,1.70)$ & $0.71(0.39,1.35)$ & & $0.87(0.51,1.51)$ & $0.69(0.30,1.60)$ & $0.86(0.41,1.88)$ & $0.45(0.21,0.98)$ & $0.49(0.25,0.97)$ & $0.98(0.53,1.97)$ \\
\hline Placebo & $1.02(0.54,1.94)$ & $0.67(0.50,0.91)$ & $0.48(0.32,0.71)$ & $0.34(0.23,0.51)$ & $0.43(0.13,1.41)$ & $0.65(0.45,0.92)$ & $0.22(0.11,0.44)$ & $1.05(0.72,1.58)$ & $0.81(0.52,1.31)$ & $1.13(0.65,1.94)$ & & $0.78(0.39,1.63)$ & $0.98(0.52,1.85)$ & $0.51(0.27,0.98)$ & $0.56(0.34,0.91)$ & $1.12(0.70,1.93)$ \\
\hline Prasugrel & $1.29(0.54,3.03)$ & $0.84(0.43,1.65)$ & $0.61(0.28,1.25)$ & $0.44(0.22,0.85)$ & $0.54(0.15,2.02)$ & $0.83(0.41,1.57)$ & $0.28(0.11,0.67)$ & $1.33(0.65,2.74)$ & $1.03(0.59,1.77)$ & $1.44(0.62,3.25)$ & $1.26(0.61,2.56)$ & & $1.24(0.52,2.94)$ & $0.65(0.27,1.56)$ & $0.72(0.31,1.60)$ & $1.42(0.71,3.00)$ \\
\hline Sarpogrelate & $1.03(0.47,2.26)$ & $0.68(0.39,1.17)$ & $0.49(0.24,0.93)$ & $0.35(0.19,0.64)$ & $0.43(0.13,1.57)$ & $0.66(0.35,1.19)$ & $0.22(0.09,0.51)$ & $1.07(0.58,2.01)$ & $0.82(0.43,1.62)$ & $1.15(0.53,2.41)$ & $1.01(0.53,1.89)$ & $0.80(0.33,1.91)$ & & $0.52(0.23,1.14)$ & $0.57(0.26,1.19)$ & $1.14(0.59,2.32)$ \\
\hline Ticagrelor & $1.96(0.90,4.30)$ & $1.29(0.74,2.27)$ & $0.94(0.45,1.79)$ & $0.67(0.36,1.22)$ & $0.83(0.24,2.94)$ & $1.27(0.66,2.30)$ & $0.42(0.18,0.98)$ & $2.02(1.09,3.89)$ & $1.57(0.80,3.09)$ & $2.20(1.01,4.61)$ & $1.92(1.01,3.59)$ & $1.52(0.64,3.66)$ & $1.89(0.87,4.17)$ & & $1.09(0.51,2.29)$ & $2.17(1.12,4.48)$ \\
\hline Ticlopidine & $1.79(0.85,3.85)$ & $1.18(0.72,1.97)$ & $0.85(0.56,1.25)$ & $0.61(0.35,1.07)$ & $0.76(0.22,2.62)$ & $1.15(0.67,1.93)$ & $0.39(0.17,0.87)$ & $1.85(1.07,3.35)$ & $1.43(0.80,2.68)$ & $2.00(1.02,3.93)$ & $1.75(1.09,2.85)$ & $1.38(0.62,3.22)$ & $1.73(0.83,3.70)$ & $0.91(0.43,1.96)$ & & $1.98(1.08,3.89)$ \\
\hline Triflusal & $0.90(0.44,1.75)$ & $0.59(0.38,0.87)$ & $0.43(0.23,0.71)$ & $0.31(0.19,0.47)$ & $0.38(0.12,1.28)$ & $0.58(0.34,0.88)$ & $0.19(0.09,0.39)$ & $0.93(0.55,1.51)$ & $0.72(0.44,1.13)$ & $1.01(0.50,1.85)$ & $0.88(0.51,1.42)$ & $0.70(0.33,1.39)$ & $0.87(0.42,1.67)$ & $0.45(0.22,0.89)$ & $0.50(0.25,0.92)$ & \\
\hline
\end{tabular}

A_1, very low dose aspirin; A_2, low to medium dose aspirin; A_3, high dose aspirin; A_C, aspirin plus clopidogrel; A_Ci, aspirin plus cilostazol; A_D, aspirin plus dipyridamole; A_Ti, aspirin plus ticagrelor. 
Table S8 Surface under the cumulative ranking curve (SUCRA) values and ranks

\begin{tabular}{|c|c|c|c|c|c|c|c|c|c|c|}
\hline \multirow{2}{*}{ Antiplatelet Regimens } & \multicolumn{2}{|c|}{ Recurrent stroke } & \multicolumn{2}{|c|}{$\begin{array}{c}\text { Recurrent ischemic } \\
\text { stroke }\end{array}$} & \multicolumn{2}{|c|}{$\begin{array}{c}\text { Composite vascular } \\
\text { events }\end{array}$} & \multicolumn{2}{|c|}{ Major bleeding } & \multicolumn{2}{|c|}{ All bleeding events } \\
\hline & SUCRA & Rank & SUCRA & Rank & SUCRA & Rank & SUCRA & Rank & SUCRA & Rank \\
\hline $\begin{array}{l}\text { Aspirin (low to medium } \\
\text { dose) }\end{array}$ & 0.2742 & 13 & 0.3496 & 11 & 0.2799 & 13 & 0.2748 & 13 & 0.4321 & 9 \\
\hline Cilostazol & 0.9199 & 1 & 0.8292 & 2 & 0.8587 & 2 & 0.8954 & 1 & 0.8075 & 3 \\
\hline Clopidogrel & 0.6928 & 5 & 0.6121 & 6 & 0.6095 & 7 & 0.4792 & 9 & 0.5981 & 7 \\
\hline Dipyridamole & 0.2228 & 14 & 0.1217 & 15 & 0.1833 & 16 & 0.8207 & 3 & 0.8433 & 2 \\
\hline Prasugrel & 0.6740 & 6 & 0.4871 & 10 & 0.6319 & 5 & 0.6740 & 6 & 0.5692 & 8 \\
\hline Ticlopidine & 0.5686 & 8 & 0.6635 & 4 & 0.6151 & 6 & 0.7604 & 4 & 0.3325 & 11 \\
\hline Triflusal & 0.3148 & 11 & 0.2910 & 13 & 0.2198 & 15 & 0.8413 & 2 & 0.8532 & 1 \\
\hline Aspirin + Cilostazol & 0.6998 & 4 & 0.5931 & 7 & 0.5131 & 9 & 0.2994 & 12 & 0.2637 & 13 \\
\hline Aspirin + Clopidogrel & 0.8249 & 2 & 0.8933 & 1 & 0.7918 & 3 & 0.0736 & 15 & 0.1003 & 15 \\
\hline Aspirin + Dipyridamole & 0.6479 & 7 & 0.6324 & 5 & 0.6735 & 4 & 0.3577 & 11 & 0.4119 & 10 \\
\hline Aspirin + Ticagrelor & 0.7238 & 3 & 0.8166 & 3 & 0.9515 & 1 & 0.0067 & 16 & 0.0181 & 16 \\
\hline Aspirin + Ticlopidine & 0.3084 & 12 & 0.5734 & 9 & 0.5142 & 8 & 0.6905 & 5 & - & - \\
\hline
\end{tabular}

A

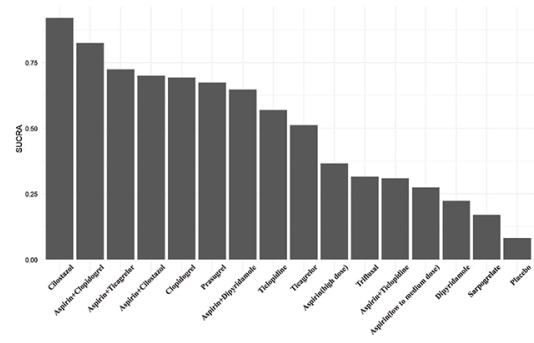

D

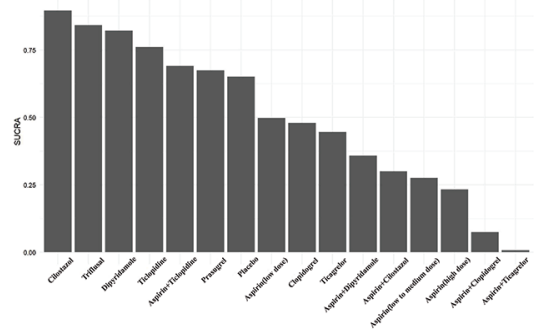

B

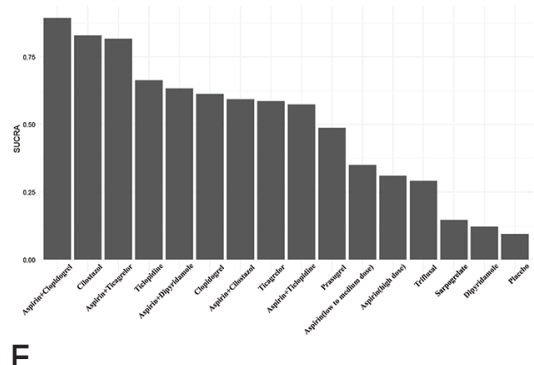

$\mathrm{E}$

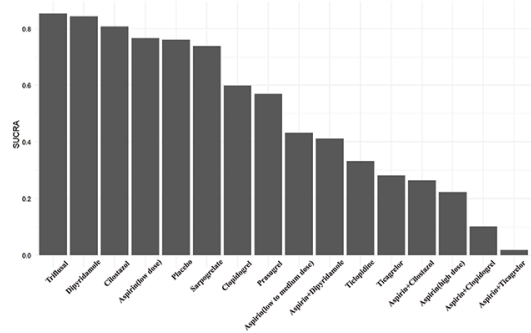

C

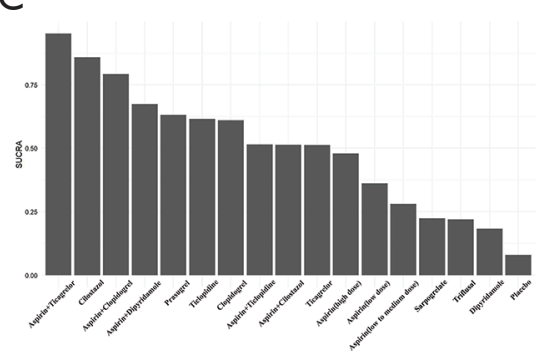

Figure S3 Surface under the cumulative ranking curve (SUCRA) rankograms for the antiplatelet regimens for (A) recurrent stroke, (B) recurrent ischemic stroke, (C) composite outcomes, (D) major bleeding events, and (E) all bleeding events. 

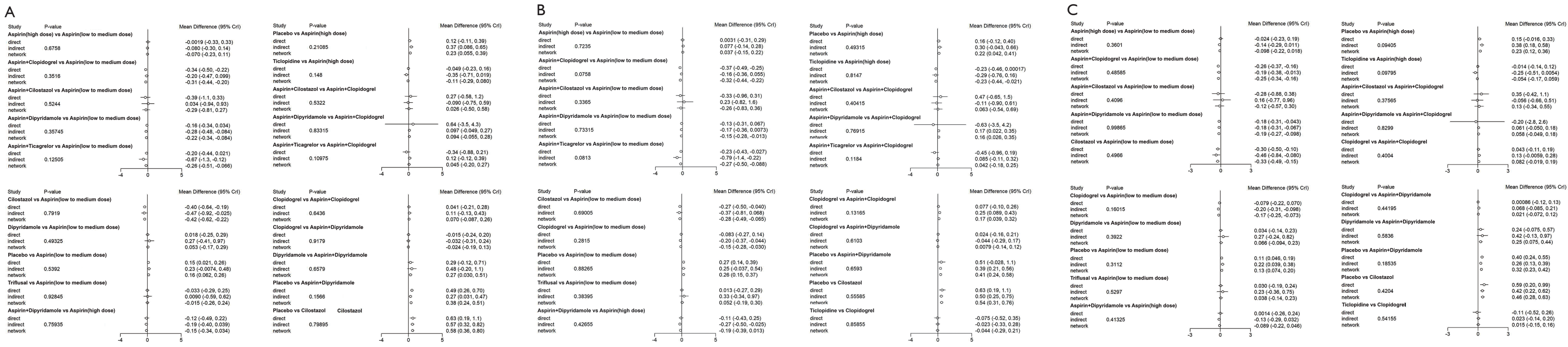

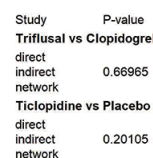
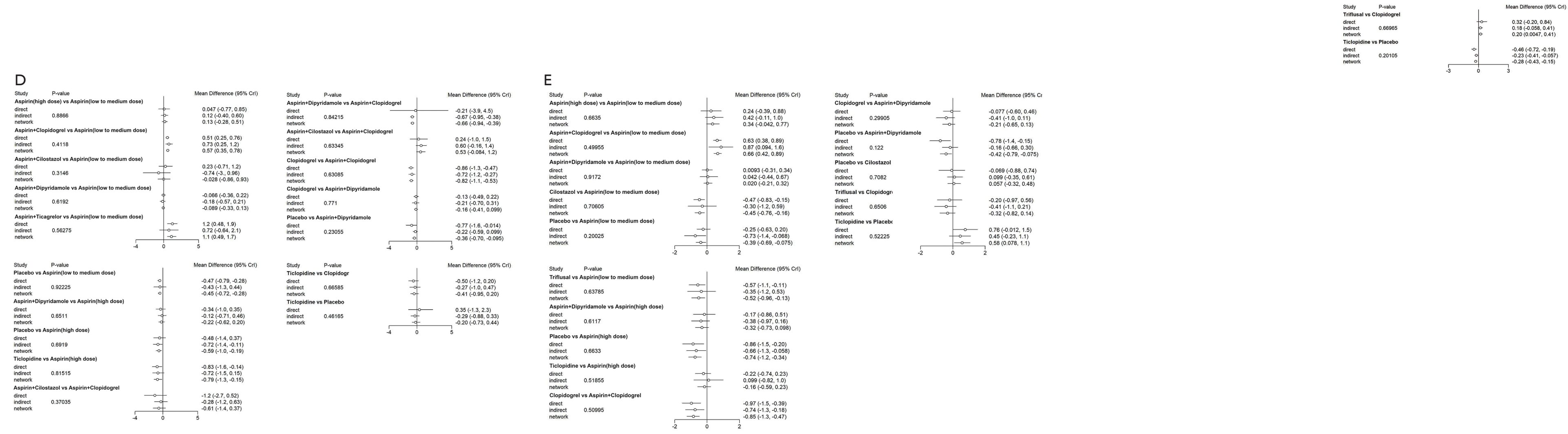

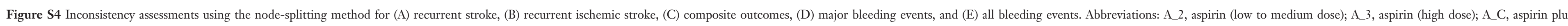

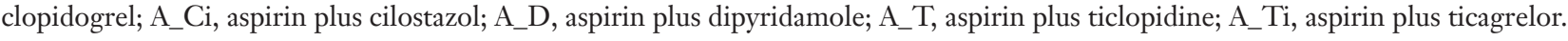



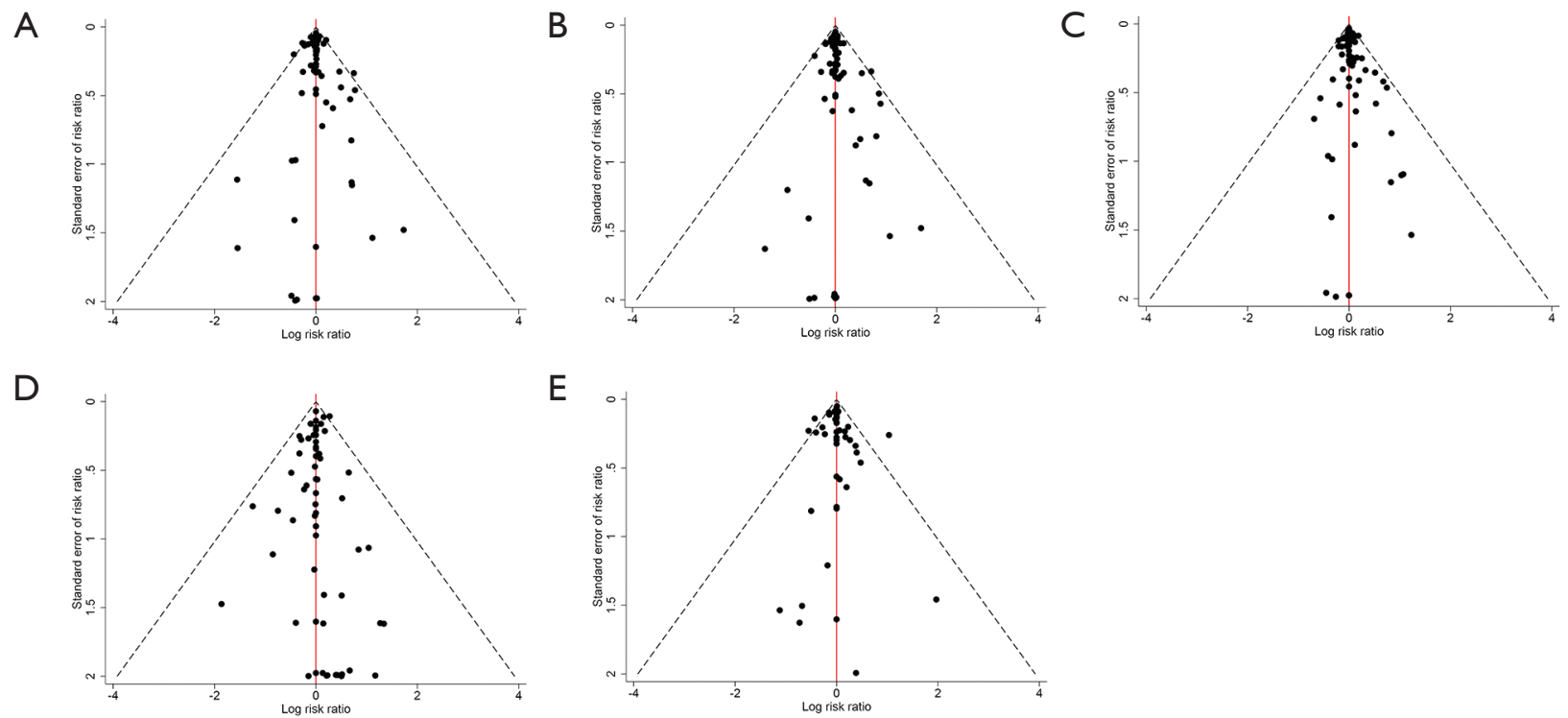

Figure S5 Funnel plots of the antiplatelet regimens of the enrolled trials. The symmetrical shape of the funnel plots demonstrates that there is no evidence of publication bias in this network meta-analysis for (A) recurrent stroke, (B) recurrent ischemic stroke, (C) composite outcomes, (D) major bleeding events, and (E) all bleeding events.

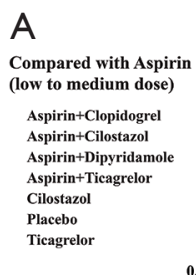

C

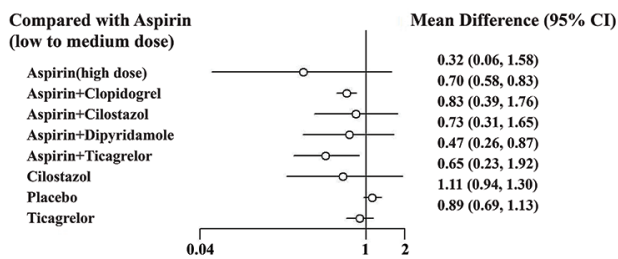

\section{B}

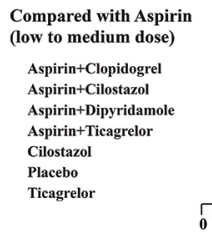

D

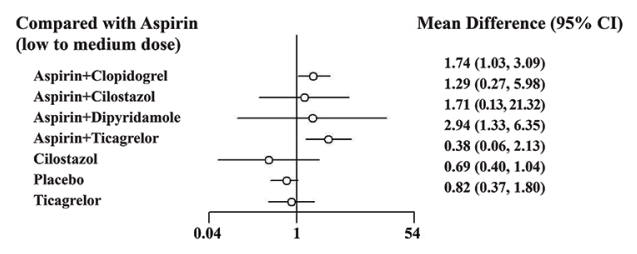

Figure S6 Forrest plots of the antiplatelet regimens compared with aspirin in the subgroup analysis ( $<72$ hours) for (A) recurrent stroke, (B) recurrent ischemic stroke, (C) composite outcomes, and (D) major bleeding events. 
A

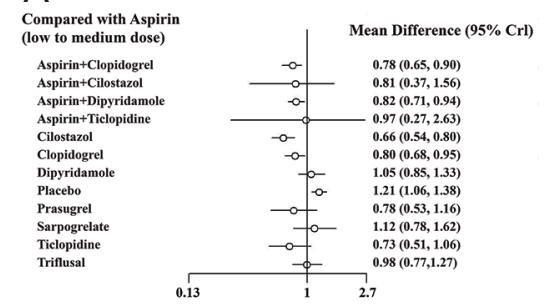

$\mathrm{D}$

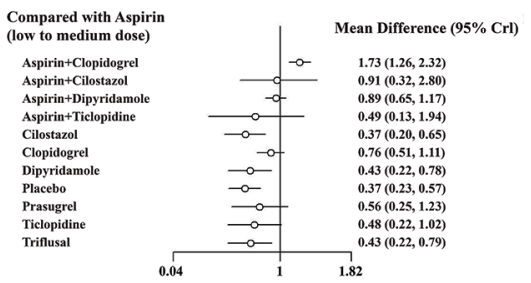

B

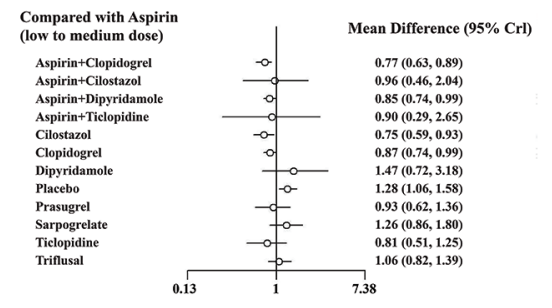

E

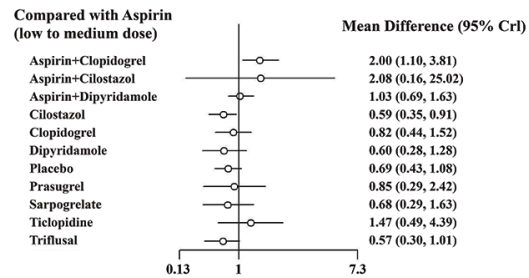

C

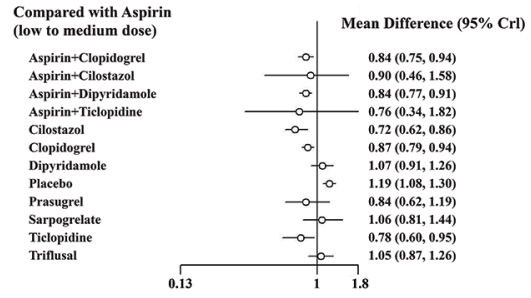

Figure S7 Forrest plots of the antiplatelet regimens compared with aspirin in the subgroup analysis ( 72 hours) for (A) recurrent stroke, (B) recurrent ischemic stroke, (C) composite outcomes, (D) major bleeding events, and (E) all bleeding events.

A

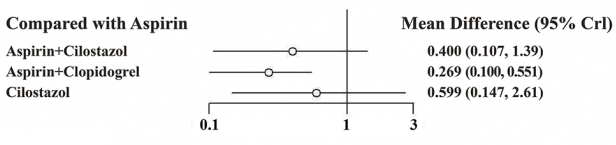

C

Compared with Aspirin Aspirin+Cilostazol
Aspirin+Clopidogrel

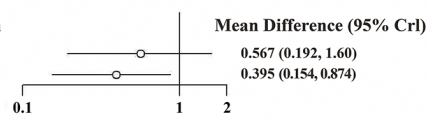

E

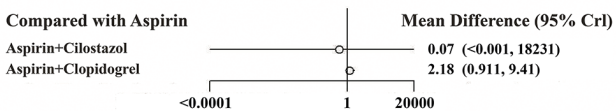

B

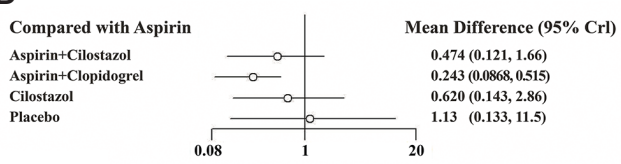

D

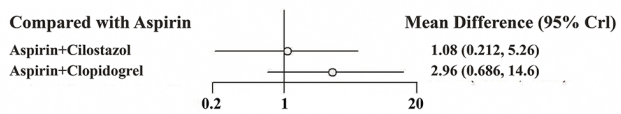

Figure S8 Forrest plots of the antiplatelet regimens compared with aspirin in the subgroup analysis for large artery atherosclerosis for (A) recurrent stroke, (B) recurrent ischemic stroke, (C) composite outcomes, (D) major bleeding events, and (E) all bleeding events. 
A

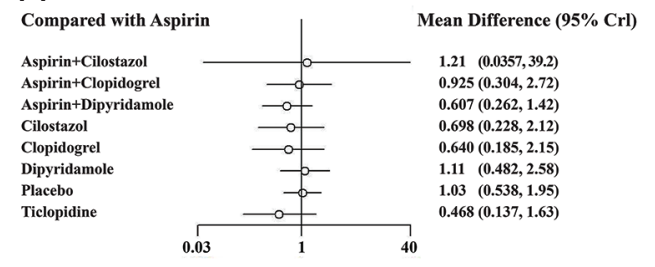

\section{C}

Compared with Aspirin

Aspirin+Cilostazol Aspirin+Clopidogrel Aspirin+Dipyridamol Cilostazol Clopidogrel Dipyridam Placebo Ticlopidine

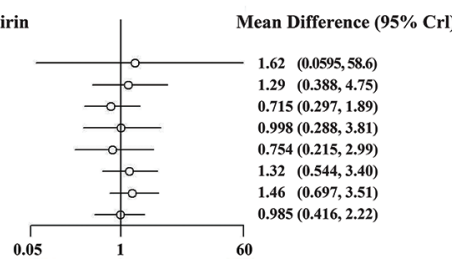

B

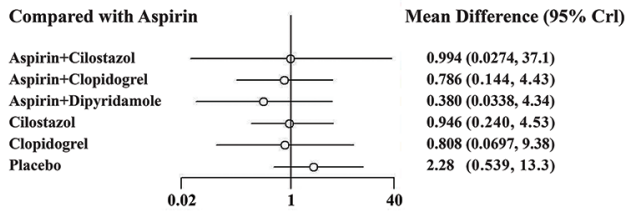

Figure S9 Forrest plots of the antiplatelet regimens compared with aspirin in the subgroup analysis for small vessel occlusion for (A) recurrent stroke, (B) recurrent ischemic stroke, and (C) composite outcomes.

A

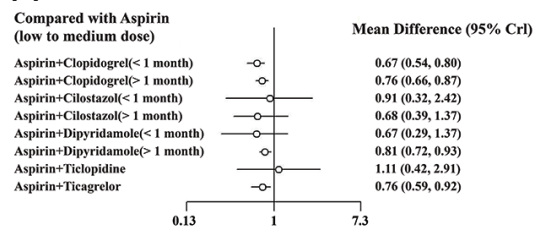

D

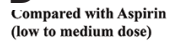
(low Aspirin+Clopidogrel $<1$ month)
Aspirin+Clopidogrel $(<1$ month $)$ Aspirin+Cilostazol(< 1 month)
Aspirin+Cilostazol(>1 month) Aspirin+Dipyridamole( $<1$ month)
Aspirin+Dipyridamole $>1$ month) Aspirin-Ticlopidie Aspirin+Ticagrelor( $<1$ month)

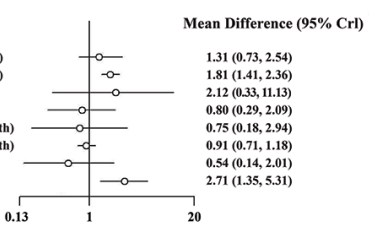

an Difference $(95 \% \mathrm{Cr}$

$1.31(0.73,2.54)$

$2.12(0.33,11.13)$

$0.75(0.18,2.94)$
$0.91(0.71,1.18$

$54(0.14,2.19)$

\section{B}

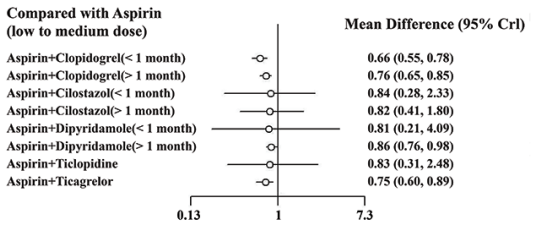

E

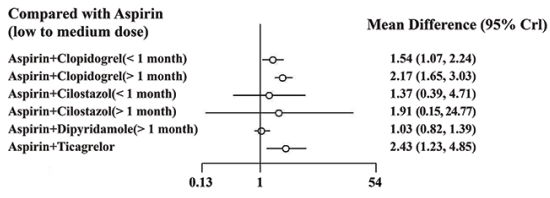

C

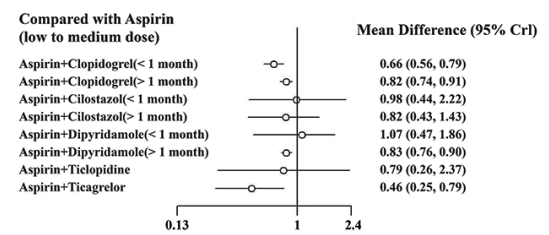

0.13

Figure S10 Forrest plots of the antiplatelet regimens compared with aspirin in the subgroup analysis for dual anti-platelet therapy, for (A) recurrent stroke, (B) recurrent ischemic stroke, (C) composite outcomes, (D) major bleeding events, and (E) all bleeding events. 